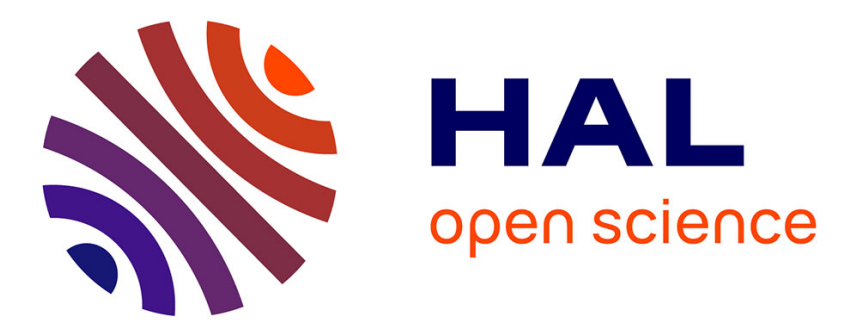

\title{
Propriétés électrochimiques de la nicotinamide et de ses dérivés en solution aqueuse: II. Étude électrochimique de l'iodure de N1-méthyl nicotinamide en solution aqueuse
}

\author{
Daniel R. Thevenot, R Buvet
}

\section{To cite this version:}

Daniel R. Thevenot, R Buvet. Propriétés électrochimiques de la nicotinamide et de ses dérivés en solution aqueuse: II. Étude électrochimique de l'iodure de N1-méthyl nicotinamide en solution aqueuse. Journal of electroanalytical chemistry and interfacial electrochemistry, 1972, pp.447-469. 10.1016/S0022-0728(72)80167-0 . hal-01179302

\section{HAL Id: hal-01179302 \\ https://hal.science/hal-01179302}

Submitted on 22 Jul 2015

HAL is a multi-disciplinary open access archive for the deposit and dissemination of scientific research documents, whether they are published or not. The documents may come from teaching and research institutions in France or abroad, or from public or private research centers.
L'archive ouverte pluridisciplinaire HAL, est destinée au dépôt et à la diffusion de documents scientifiques de niveau recherche, publiés ou non, émanant des établissements d'enseignement et de recherche français ou étrangers, des laboratoires publics ou privés. 


\title{
PROPRIÉTÉS ÉLECTROCHIMIQUES DE LA NICOTINAMIDE ET DE SES
} DÉRIVÉS EN SOLUTION AQUEUSE

\author{
II. ÉTUDE ÉLECTROCHIMIQUE DE L'IODURE DE N ${ }_{1}$-MÉTHYL \\ NICOTINAMIDE EN SOLUTION AQUEUSE*
}

\section{THÉVENOT et R. BUVET}

Laboratoire d'Energétique Biochimique, Centre Multidisciplinaire, Université Paris-Val de Marne, Avenue du Général de Gaulle, 94, Créteil (France)

(Reçu le 14 décembre 1971; en forme revisée le 27 avril 1972)

Depuis une vingtaine d'années les sels de $\mathrm{N}_{1}$-alkyl pyridinium et en particulier l'iodure de $\mathrm{N}_{1}$-méthyl nicotinamide ont été utilisés comme composés modèles du nicotinamide-adénine-dinucléotide. L'analyse critique de l'ensemble des données disponibles relatives aux propriétés électrochimiques de ces dérivés ${ }^{35}$ montre qu'il subsiste encore des lacunes, des difficultés et des imprécisions. Les expériences antérieures ${ }^{28-30}$ sont insuffisantes puisque dans la plupart des cas seule l'influence du pH de la solution a été en partie étudiée et que les effets des autres paramètres expérimentaux (concentration du réactif, nature des tampons, température, nature de l'électrode...) ont été trop négligés. Les mécanismes de réactions proposés pour la réduction électrochimique de ces dérivés comme par exemple la formation de dimère ${ }^{31}$ reposent essentiellement sur les résultats de macroélectrolyses à potentiel contrôlés. On sait que cette technique peut conduire à des interprétations erronées car très fréquemment l'étape réactionelle qui fixe la valeur du potentiel ne représente qu'une partie de la transformation globale. Enfin les hypothèses interprétatives ont été souvent émises de façon incohérente et sont parfois thermodynamiquement incompatibles ${ }^{35}$.

Nous avons par conséquent repris l'étude électrochimique d'un sel de $\mathrm{N}_{1}$ méthyl nicotinamide en nous attachant à l'analyse détaillée de l'influence des différents facteurs expérimentaux sur les courbes intensité-potentiel relevées ${ }^{1}$.

\section{RÉDUCTION DE SOLUTIONS AQUEUSES TRÈS DILUÉES DE L'IODURE DE $\mathrm{N}_{1}$-MÉTHYL NICOTINAMIDE SUR GOUTTE DE MERCURE EN STILLATION COMMANDÉE: INFLUENCE DU pH DE LA SOLUTION}

Si la concentration d'iodure de $\mathrm{N}_{1}$-méthyl nicotinamide est inférieure à environ $10^{-3} \mathrm{M}$, tous les phénomènes observés restent qualitativement comparables lorsque cette concentration est modifiée. Pour mettre en évidence l'influence du $\mathrm{pH}$ sur la position et la hauteur des vagues polarographiques en solution diluée, nous

* Partie de la thèse de doctorat d'Etat ès-Sciences Physiques soutenue par Daniel Thévenot le 14 Octobre 1971 à Paris. No. CNRS : A.O. 6164. 
comparons les résultats obtenus pour une même concentration de réactif par exemple $10^{-4} \mathrm{M}$.

La réduction polarographique de l'iodure de $\mathrm{N}_{1}$-méthyl nicotinamide en solution très diluée $\left(10^{-4} \mathrm{M}\right)$ fait toujours apparaitre deux vagues successives, mais les caractéristiques de celles-ci sont très différentes suivant que le $\mathrm{pH}$ de la solution est inférieur ou supérieur à environ 3 (Fig. 1).

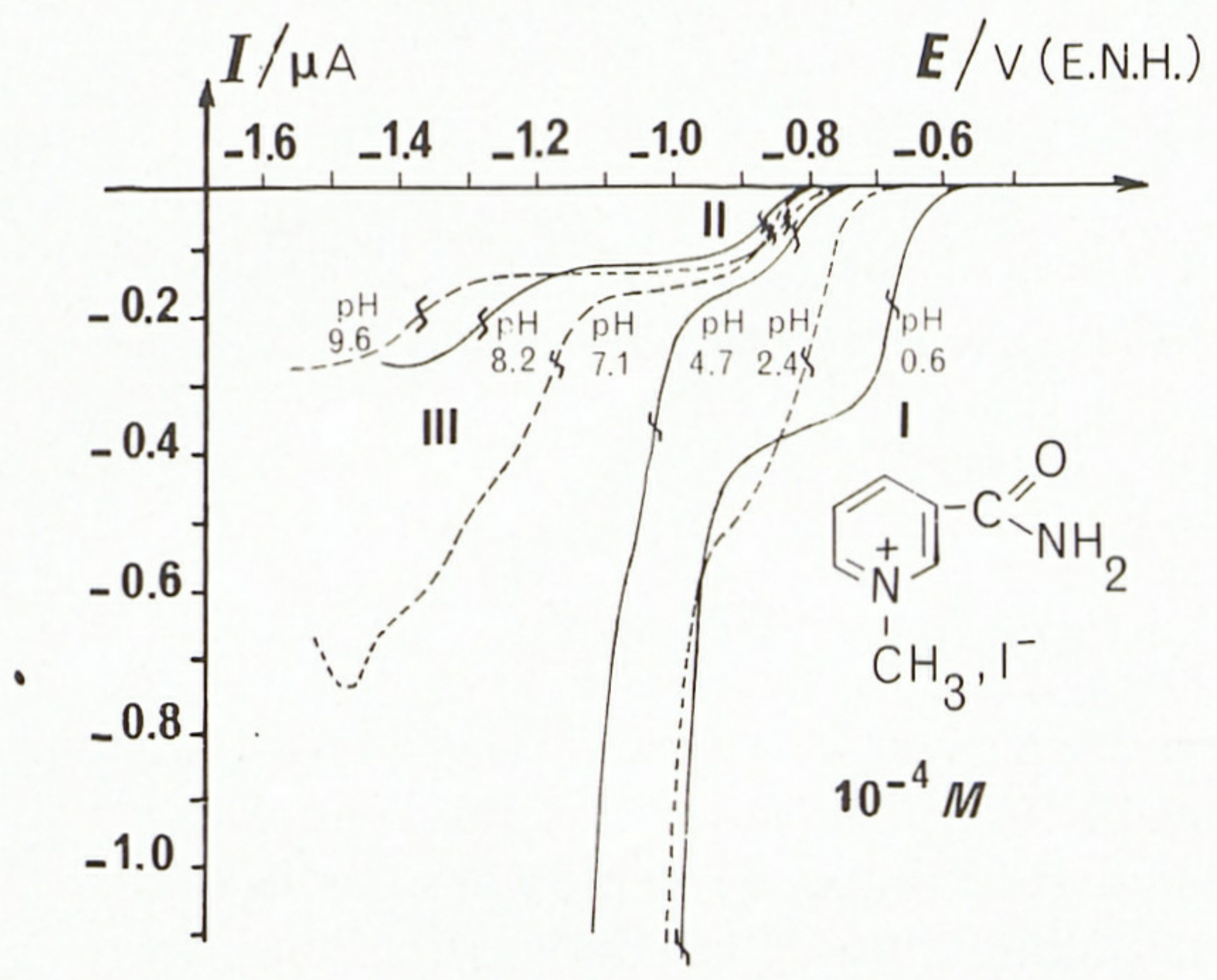

Fig. 1. Réduction de solutions aqueuses $10^{-4} \mathrm{M}$ d'iodure de $\mathrm{N}_{1}$-méthyl nicotinamide sur goutte de mercure à stillation commandée: pH 0.56 ( $\mathrm{HCl}), 2.41$ (glycine), 4.68 (acétate), 7.15 (imidazole), 8.25 (tris) et 9.57 (borate). La vague I' correspond à un simple épaulement le long du mur de réduction des protons; nous n'avons fait figurer sur ce dessin qu'une partie de la vague I' car celle-ci présente une hauteur 4-10 fois plus grande que celle de la vague I. La position des $E_{\frac{1}{2}}$ est notée $(\sim)$. Conditions polarographiques: capillaire no. $10,35.0 \mathrm{~cm}$ de mercure, temps de goutte imposé $0.50 \mathrm{~s}$, balayage des potentiels à $250 \mathrm{mV} \mathrm{min}^{-1}$, $25.0 \pm 0.2^{\circ} \mathrm{C}$.

En milieu très acide une vague I est suivie d'une vague I', de hauteur bien supérieure, qui est souvent assez mal formée car proche du mur de réduction des protons sur mercure. Les vagues I et I' se déplacent toutes deux vers les potentiels plus négatifs lorsque le $\mathrm{pH}$ de la solution augmente.

Dans les solutions de $\mathrm{pH}$ compris entre 3 et 10 il existe également deux vagues de réduction, que nous noterons II et III. La vague II ne dépend ni pour sa position, ni pour sa hauteur du $\mathrm{pH}$ de la solution. Sa hauteur est à peu près la moitié de la hauteur de la vague I en milieu très acide. Ce que nous appelons vague III corresponden fait lorsque le $\mathrm{pH}$ est compris entre 3 et 8 à un ensemble de manifestations mal définies. Il s'agit de l'apparition d'un épaulement le long soit d'un mur de réduction, soit d'un maximum polarographique qui eux-mêmes n'existent pas en absence de réactif (Fig. 1, pH 4.7 en 7.1). Par contre, entre pH 8 et 10 la vague III apparaît très nettement et présente une hauteur sensiblement égale à celle de la vague II. La raison qui nous a amené à ranger les manifestations observées entre $\mathrm{pH} 3$ et 10 sous une même dénomination est que les $E_{\frac{1}{2}}$ relevés, tant pour les épaulements que pour les vagues, varient continûment avec le pH (Fig. 2).

La Fig. 2 présente les variations des $E_{\frac{1}{2}}$ des vagues ainsi définies avec le $\mathrm{pH}$ de la solution de l'iodure de $\mathrm{N}_{1}$-méthyl nicotinamide $10^{-4} \mathrm{M}$. Ces variations sont linéaires et ont pour expression: 
Vague I $\quad E_{\frac{1}{2}}=(-633-74 \mathrm{pH}) \pm 10 \mathrm{mV} / \mathrm{ENH}$

Vague I' $\quad E_{\frac{1}{2}}=(-970-45 \mathrm{pH}) \pm 5 \mathrm{mV} / \mathrm{ENH}$

Vague II $\quad E_{\frac{1}{2}}=(-830) \pm 30 \mathrm{mV} / \mathrm{ENH}$

Vague III $E_{\frac{1}{2}}=(-750-60 \mathrm{pH}) \pm 30 \mathrm{mV} / \mathrm{ENH}$

Un travail récent sur la vague $\mathrm{I}^{36}$ a montré qu'en augmentant considérablement le nombre de tampons et de $\mathrm{pH}$ étudiés, les valeurs des potentiels de demi-vague ne vérifient la première relation qu'à $\pm 30 \mathrm{mV}$ près et que finalement $\Delta E_{\frac{1}{2}} / \Delta \mathrm{pH}$ n'est connu qu'assez approximativement: $80 \pm 20 \mathrm{mV}$.

La valeur de $E_{\frac{1}{2}}$ de la vague II obtenue dans le tampon tris à $\mathrm{pH} 8.25$ est exceptionellement faible ( $45 \mathrm{mV}$ au-dessous du segment de droite). La valeur de $E_{\frac{1}{2}}$ de la vague III obtenue dans le tampon borate à $\mathrm{pH} 9.58$ est également exceptionellement faible $(50 \mathrm{mV}$ au-dessous du segment de droite). Ces deux valeurs n'ont pas été prises en considération pour définir les relations algébriques précédentes.
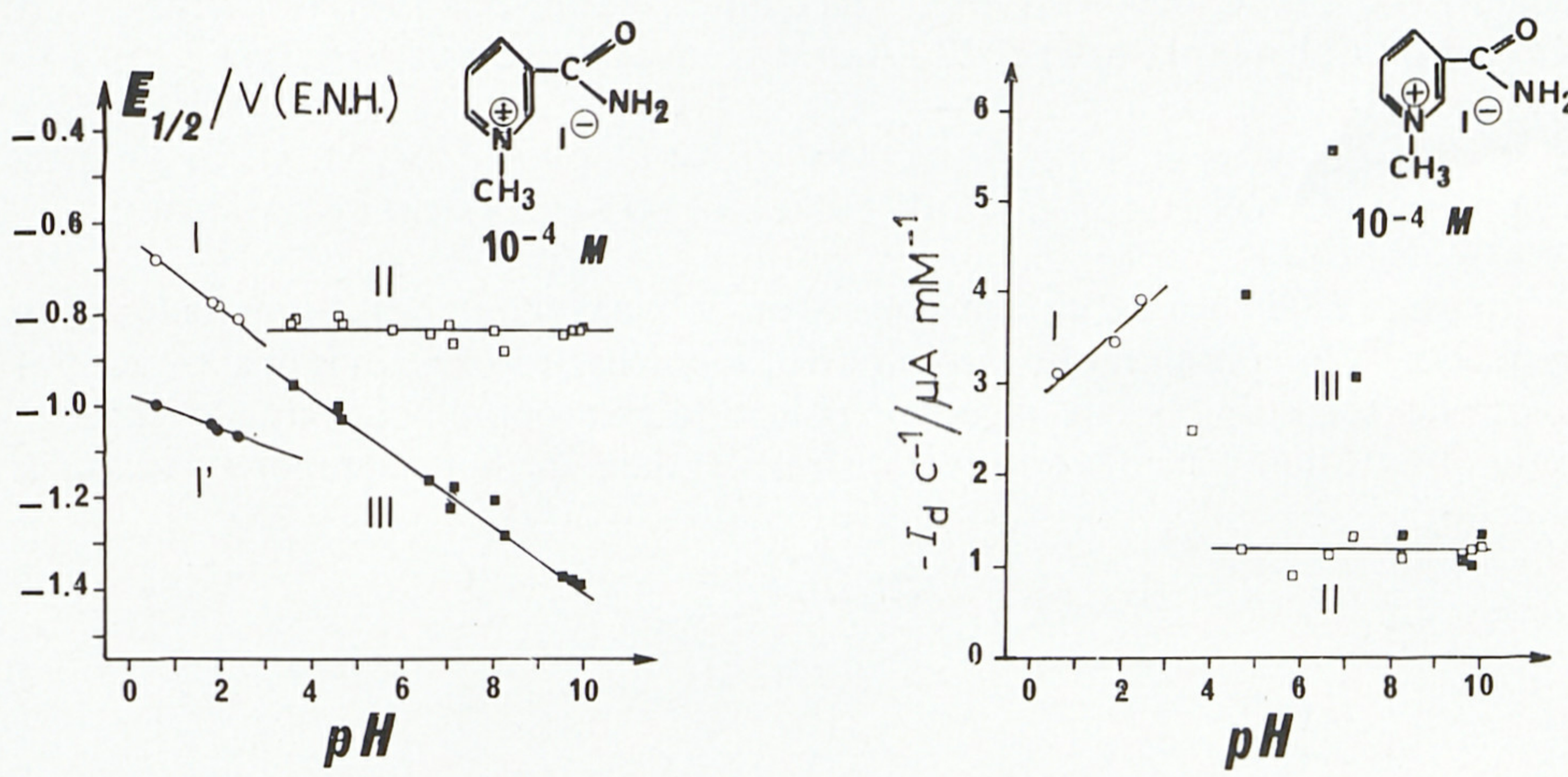

Fig. 2. Réduction de solutions aqueuses $10^{-4} M$ d'iodure de $\mathrm{N}_{1}$-méthyl nicotinamide sur goutte de mercure à stillation commandée. Influence du pH sur les potentiels de demi-vague. $(O)$ vague $\mathrm{I},(\bullet)$ vague $\mathrm{I}^{\prime}$. ( $\square$ ) vague II, (圆) vague III. Conditions polarographiques: capillaire no. $10,35.0 \mathrm{~cm}$ de mercure, temps de

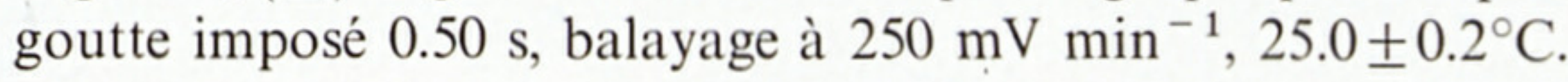

Fig. 3. Réduction de solutions aqueuses $10^{-4} \mathrm{M}$ d'iodure de $\mathrm{N}_{1}$-méthyl nicotinamide sur goutte de mercure à stillation commandée. Influence du $\mathrm{pH}$ sur les hauteurs relatives des vagues. Conditions, voir Fig. 2. La hauteur de la vague I' étant considérablement supérieure à celle des vagues I, II et III, nous n’avons pas représenté dans ce dessin les points correspondant à cette vague I'.

De façon semblable la Fig. 3 présente la variation avec le $\mathrm{pH}$ des courants limites relatifs des vagues, pour des solutions $10^{-4} \mathrm{M}$. Les conditions expérimentales polarographiques utilisées sont identiques pour tous les relevés : temps de goutte libre $9.5 \pm 0.5 \mathrm{~s}$, temps de goutte imposé $0.50 \mathrm{~s}$, débit de mercure $m=0.86 \pm 0.03 \mathrm{mg} \mathrm{s}^{-1}$, $25.0 \pm 0.2^{\circ} \mathrm{C}$, ces données étant obtenues avec un capillaire référencé 10 pour une hauteur de $35.0 \mathrm{~cm}$ de mercure. Les courants limites obtenus pour la vague II dépendent peu du pH de la solution (écarts inférieurs à $\pm 10 \%$ ), à l'exception de la valeur relevée à pH 3.6 dans le tampon formiate pour lequel les vagues II et III sont mal sé- 
parées. Les courants limites varient davantage pour la vague $I( \pm 12 \%)$ et varient surtout de façon importante pour les secondes vagues de réduction I' et III tant en milieu très acide (vague $\mathrm{I}^{\prime}$ ) qu'à $\mathrm{pH}$ compris entre 3 et 8 (vague III); ces deux dernières vagues sont en effet déformées car situées le long d'une courbe courant-tension de forme mal définie.

En résumé pour

- la vague I : $-I_{\mathrm{lim}} / c=3.0 \pm 0.4 \mu \mathrm{A} \mathrm{m} M^{-1}$

- la vague $\mathrm{I}^{\prime}: 13.6 \leqslant-I_{\lim } / c \leqslant 29.2 \mu \mathrm{A} \mathrm{m} M^{-1}$

- la vague II : $-I_{\mathrm{lim}} / c=1.20 \pm 0.12 \mu \mathrm{A} \mathrm{m} M^{-1}$

- la vague III : $\mathrm{pH} \leqslant 8 \quad 3.1 \leqslant-I_{\lim } / c \leqslant 5.6 \mu \mathrm{A} \mathrm{m} M^{-1}$

$$
\mathrm{pH} \geqslant 8-I_{\lim } / c=1.20 \pm 0.12 \mu \mathrm{A} \mathrm{m} M^{-1}
$$

Le pic polarographique que nous avons observé en milieu neutre est situé à $-1570 \pm 20 \mathrm{mV} / \mathrm{ENH}$ et présente une hauteur considérable à l'échelle des autres vagues: $-I_{\mathrm{p}} / c$ est égal à $63 \mu \mathrm{A} \mathrm{m} M^{-1}$ en tampon citrate $(\mathrm{pH} 5.78), 14 \mu \mathrm{A} \mathrm{m} M^{-1}$ en tampon phosphate ( $\mathrm{pH}$ 6.61) et $27 \mu \mathrm{A} \mathrm{m} M^{-1}$ en tampon imidazole ( $\left.\mathrm{pH} 7.15\right)$.

\section{INFLUENCE DE LA CONCENTRATION DE L'IODURE DE $\mathrm{N}_{1}$-MÉTHYL NICOTINAMIDE} SUR SA RÉDUCTION SUR GOUTTE DE MERCURE EN STILLATION COMMANDÉE

A la différence de ce que nous avons observé pour la nicotinamide ${ }^{2}$ et la pyrimidine ${ }^{3}$, la concentration d'iodure de $\mathrm{N}_{1}$-méthyl nicotinamide n'a en général pas d'effet sur le nombre de vagues de réduction polarographique. Une exception à cette observation générale est toutefois relevée dans quelques tampons très acides
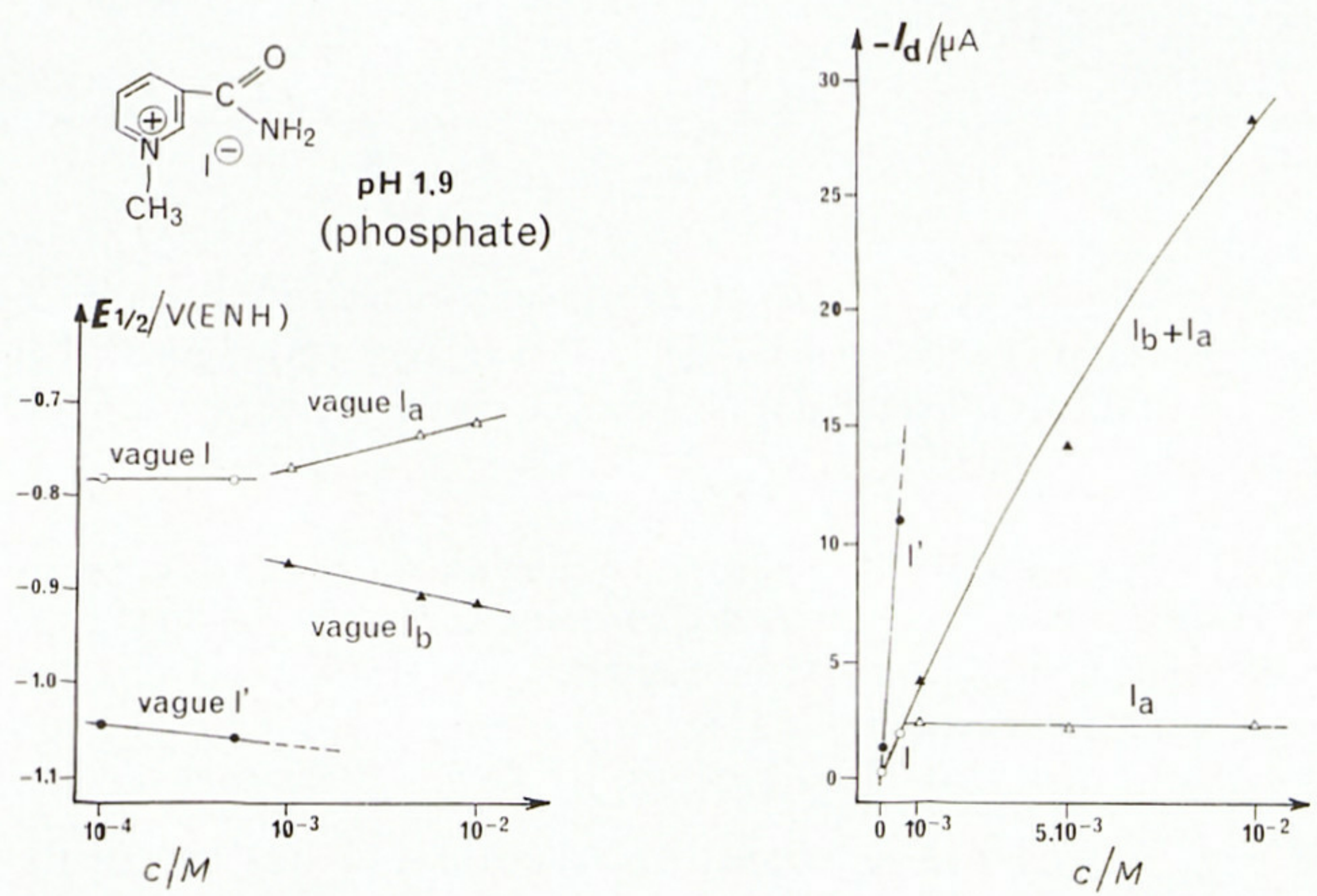

Fig. 4. Influence de la concn. de l'iodure de $\mathrm{N}_{1}$-méthyl nicotinamide sur sa réduction sur goutte de mercure en stillation commandée. Comportement particulier relevé à pH 1.86 dans une solution tampon contenant $\mathrm{H}_{3} \mathrm{PO}_{4}$ et $\mathrm{KH}_{2} \mathrm{PO}_{4}$ à la concn. $0.5 \mathrm{M}$. Effet de la concn. de réactif sur le nombre et le potentiel des vagues.

Fig. 5. Influence de la concn. de l'iodure de $\mathrm{N}_{1}$-méthyl nicotinamide sur sa réduction sur goutte de mercure en stillation commandée. Comportement particulier relevé à pH 1.86 dans une solution tampon contenant $\mathrm{H}_{3} \mathrm{PO}_{4}$ et $\mathrm{KH}_{2} \mathrm{PO}_{4}$ à la concn. $0.5 \mathrm{M}$. Effet de la concn. de réactif sur le nombre et la hauteur des vagues. 
( $\mathrm{pH}$ voisin de 2) comme par exemple le tampon orthophosphate à $\mathrm{pH} 1.86$ (Figs. 4 et 5). Dans une solution contenant $\mathrm{H}_{3} \mathrm{PO}_{4} 0.5 \mathrm{M}$ et $\mathrm{KH}_{2} \mathrm{PO}_{4} 0.5 \mathrm{M}$, la vague I se dédouble en deux parties $\mathrm{I}_{\mathrm{a}}$ et $\mathrm{I}_{\mathrm{b}}$ si la concentration de réactif est supérieure à environ $7 \times 10^{-4} M$; la vague $\mathrm{I}_{\mathrm{a}}$ présente dans les conditions polarographiques utilisées (capillaire 10, $35.0 \mathrm{~cm}$ de mercure, temps de goutte imposé $0.5 \mathrm{~s}$ ) un courant de $2.3 \pm 0.1$ $\mu \mathrm{A}$ indépendant de la concentration de réactif.

Dans tous les milieux où la vague I a été observée, $\mathrm{pH}$ inférieurs à 3 , celle-ci a unehauteur qui n'est qu'approximativement proportionnelle à la concentration de réactif ; la hauteur relative $-I_{\text {lim }} / c$ diminue de $30-50 \%$ lorsque $c$ passe de $10^{-4}$ à $10^{-2} \mathrm{M}$ bien qu'aucune décomposition du réactif n'ait été observée dans ces milieux acides. La deuxième vague, vague I', présente une hauteur qui n'est également qu'approximativement proportionelle à la concentration de réactif; sa hauteur relative $-I_{\mathrm{lim}} / c$ dépend fortement du tampon tout en restant beaucoup plus grande que celle de la vague $\mathrm{I}: 15.5 \pm 2 \mu \mathrm{A} \mathrm{m} M^{-1}$ à $\mathrm{pH} 0.6$ (acide chlorhydrique), $13-22 \mu \mathrm{A} \mathrm{m} M^{-1}$ à $\mathrm{pH} 1.8$ (phosphate) et $29.3 \pm 0.1 \mu \mathrm{A} \mathrm{m} M^{-1}$ à $\mathrm{pH} 2.4$ (glycine). De façon analogue, le pic observé à potentiel très cathodique en milieu neutre présente une importante hauteur relative $-I_{\text {lim }} / c$ qui varie beaucoup avec le $\mathrm{pH}$ et la concentration de réactif (14-92 $\left.\mu \mathrm{A} \mathrm{m}^{-1}\right)$.

Les vagues II et III ont des hauteurs proportionnelles à la concentration de l'iodure de $\mathrm{N}_{1}$-méthyl nicotinamide lorsque celle-ci varie entre $10^{-5}$ et $10^{-2} \mathrm{M}$. Ceci est observé en vérifiant que $\log \left(-I_{\lim }\right)$ varie bien linéairement avec $\log c$, la pente restant en général assimilable à $1(1.00 \pm 0.05)$, puis en comparant les valeurs de $-I_{\text {lim }} / c$ pour les différentes valeurs de $c$. Pour les vagues II et III, c'est-à-dire pour des solutions de $\mathrm{pH}$ supérieur à 3 , les hauteurs relatives $-I_{\mathrm{lim}} / c$ varient généralement au plus de $5-10 \%$ avec $c$, ce qui reste compatible avec une relation linéaire entre $I_{\text {lim }}$ et $c$ compte tenu de la précision des mesures de courants limites. Cependant en tampons tormiate $(\mathrm{pH} 3.6)$ pour la vague II et acétate $(\mathrm{pH} 4.7)$ pour la vague III, les variations de hauteurs relatives sont plus importantes; $-I_{1 \mathrm{im}} / c$ varie respectivement de 2.5 à 1.3 $\mu \mathrm{A} \mathrm{m} M^{-1}$ entre $10^{-4}$ et $10^{-2} M$ à $\mathrm{pH} 3.6$ pour la vague II et de 5 à $2 \mu \mathrm{A} \mathrm{m} M^{-1}$ entre $10^{-3}$ et $10^{-2} \mathrm{M}$ à $\mathrm{pH} 4.7$ pour la vague III.

Alors qu'en milieu très acide la position de la vague I ne dépend pas de la concentration de réactif, dans le domaine des $\mathrm{pH}$ de 3-10 les deux vagues II et III se déplacent en potentiel de façon significative lorsque la concentration de ce réactif passe de $10^{-4}$ à $10^{-2} M$ (Fig. 6).

Le comportement de la vague II est différent suivant que la concentration de réactif est inférieure ou supérieure à environ $10^{-3} \mathrm{M}$. En solution plus diluée que $10^{-3}$ $M, E_{\frac{1}{2}}$ augmente de $19 \pm 1 \mathrm{mV}$ par décade de concentration, alors qu'en solution plus concentrée $E_{\frac{1}{2}}$ reste sensiblement constant. Ce comportement est très semblable à celui observé pour la première vague de réduction de la pyrimidine en milieu acide ${ }^{3}$. Les valeurs de $E_{\frac{1}{2}}$ relevées dans les solutions de tampon tris à pH 8.25 (Fig. 6) s'écartent légèrement de ce comportement; elles augmentent de $32 \mathrm{mV}$ par décade de concentration. De plus dans les solutions d'orthophosphate ( $\mathrm{pH}$ 6.61) la vague II a un potentiel de demi-vague qui augmente de -840 à $-800 \mathrm{mV} / \mathrm{ENH}$ entre $10^{-4}$ et $5 \times 10^{-4}$ $M$, puis diminue de $10 . \mathrm{mV}$ par décade de concentration entre $5 \times 10^{-4}$ et $10^{-2} \mathrm{M}$.

Le comportement de la vague III diffère suivant que le $\mathrm{pH}$ de la solution est supérieur ou inférieur à environ 5. Dans les solutions tampons contenant du formiate et de l'acétate, respectivement à $\mathrm{pH} 3.56$ et $4.68, E_{\frac{1}{2}}$ est indépendant de la concen- 


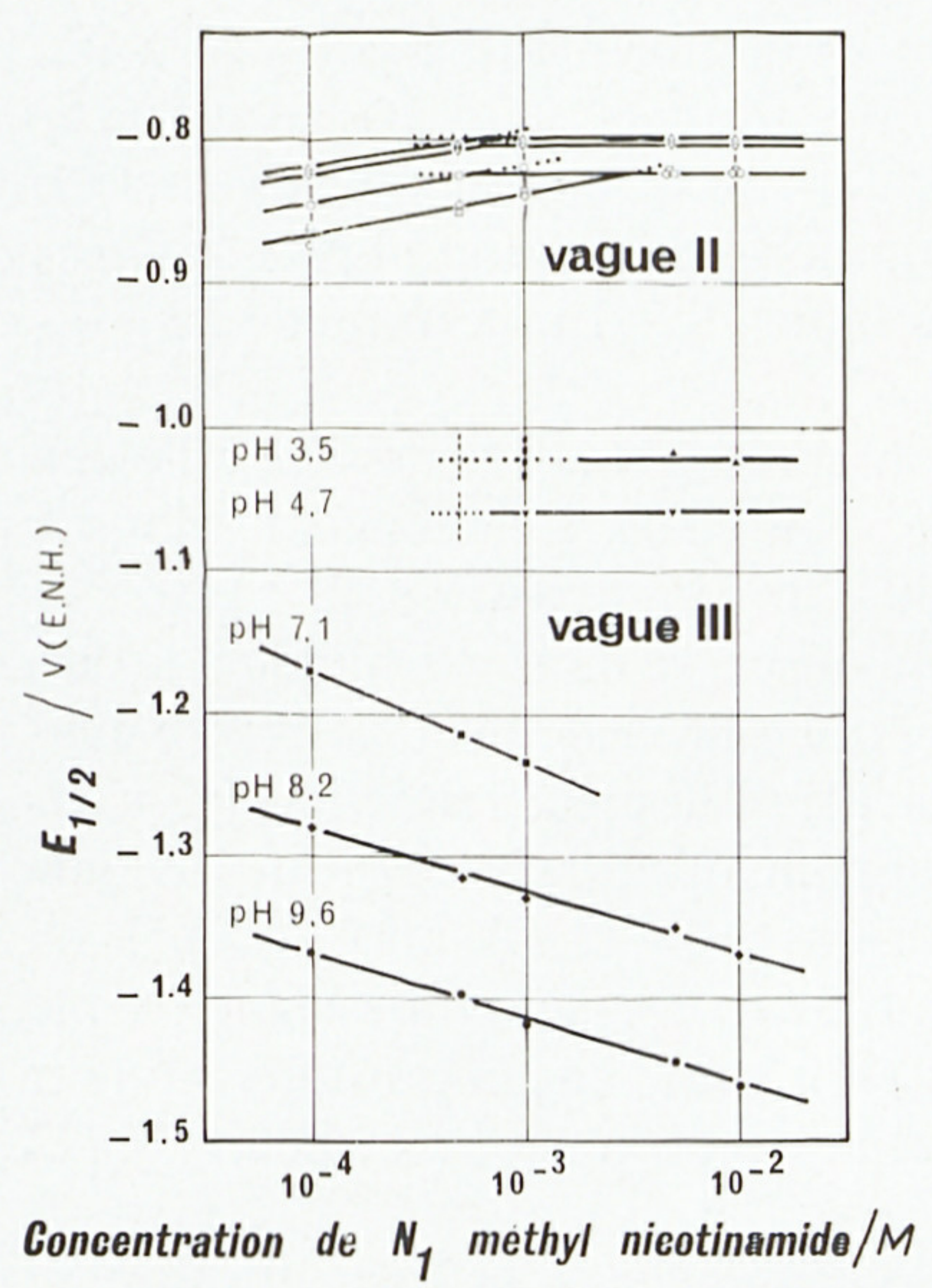

Fig. 6. Influence de la concn. de l'iodure de $\mathrm{N}_{1}$-méthyl nicotinamide sur sa réduction sur goutte de mercure en stillation commandée. Comportement des vagues II et III dans les tampons suivants : $(\Delta$ et $\mathbf{\Delta})$ formiate $\mathrm{pH} 3.56,(\nabla$ et $\boldsymbol{\nabla})$ acétate $\mathrm{pH} 4.68$, ( $\square$ et $\mathbf{\nabla})$ imidazole $\mathrm{pH} 7.15(\diamond$ et $\diamond)$ tris $\mathrm{pH} 8.25$, $(\bigcirc$ et $\odot)$ borate $\mathrm{pH}$ 9.57. Influence de la concentration du réactif sur les potentiels de demi-vague.

tration, alors que dans les solutions tampons contenant de l'imidazole, du tris, des borates, des carbonates et du glycinate, respectivement à pH 7.15, 8.25, 9.57, 9.82 et 9.97, $E_{\frac{1}{2}}$ diminue respectivement de $64,44,44,40$ et $43 \mathrm{mV}$ par décade de concentration. Puisque c'est seulement aux pH supérieurs à 8 que la vague III est entièrement formée (elle est alors distante de plus de $200 \mathrm{mV}$ du mur de réduction du solvant), nous retiendrons son comportement dans les tampons tris, borate, carbonate et glycinate, comme le plus significatif, alors $\Delta E_{\frac{1}{2}} / \Delta \log c=-43 \pm 3 \mathrm{mV}$.

Le glissement du potentiel de demi-vague des vagues II et III, lorsque la concentration de pyrimidine varie, révèle l'existence dans les mécanismes de réaction correspondant à ces deux vagues.

— soit d'étapes de réaction, d'ordre différent de l'unité, qui limiteraient la vitesse de transfert des électrons,

- soit de réactions d'oxydo-réduction réversibles conduisant à des produits en nombre différent des réactifs, les composés à potentiel chimique constant (solvant, solides, constituants du tampon) n'interviennent pas dans ce décompte ${ }^{4}$.

3. INFLUENCE DES PARAMÈTRES EXPÉRIMENTAUX SUR LA RÉDUCTION

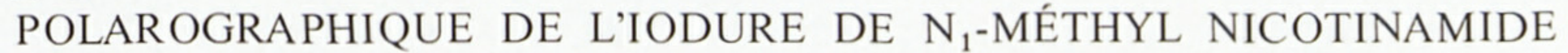

\subsection{Effet de la nature du tampon}

L'analyse des résultats présentés dans les Figs. 2 et 3 (influence du pH sur $E_{\frac{1}{2}}$ et sur $I_{\text {lim }}$ si $c=10^{-4} M$ ) ainsi que dans la Fig. 6 (influence de la concentration sur $\left.E_{\frac{1}{2}}\right)$ montre que ces résultats sont en général indépendant de la nature du tampon qui fixe le $\mathrm{pH}$ de la solution. 
Il nous semble que si les vagues I' et III ont des hauteurs relatives $-I_{\text {lim }} / c$ qui dépendent beaucoup du milieu et de la concentration de réactif, ceci est sans doute dû au fait qu'elles sont situées le long d'une courbe courant-tension de forme exponentielle qui elle-même dépend souvent de la nature du tampon et de la concentration de réactif : courbe de fond pour la vague I' et flanc d'un pic polarographique pour la vague III.

\subsection{Effet de la fréquence de chute des gouttes de mercure}

Le temps de goutte joue en polarographie un rôle inverse de celui de la vitesse de balayage des potentiels en voltamétrie sur électrode immobile. Son influence sur le potentiel de demi-vague est utilisée pour déterminer l'existence de réaction chimique consécutive ou simultanée à la réaction de transfert d'électrons ${ }^{5}$. Son influence sur la hauteur de la vague étudiée permet de savoir si le processus limitant le courant est la diffusion du réactif, une cinétique de réaction ou une adsorption irréversible sur l'électrode.

En maintenant constant le débit de mercure, c'est-à-dire en pratique la hauteur de la colonne de mercure au-dessus du capillaire, nous avons étudié l'influence du temps de goutte imposé $\tau$ tant sur le potentiel de demi-vague que sur la hauteur de toutes les vagues de réduction de l'iodure de $\mathrm{N}_{1}$-méthyl nicotinamide.

Pour un système électrochimique réversible comme $\mathrm{Tl}^{0} / \mathrm{Tl}^{+}$ou $\mathrm{Cd}^{0} / \mathrm{Cd}^{2+}$ la fréquence de chute de gouttes de mercure n'a aucun effet sur le potentiel de demivague. Dans le cas de la réduction de l'iodure de $\mathrm{N}_{1}$-méthyl nicotinamide nous avons

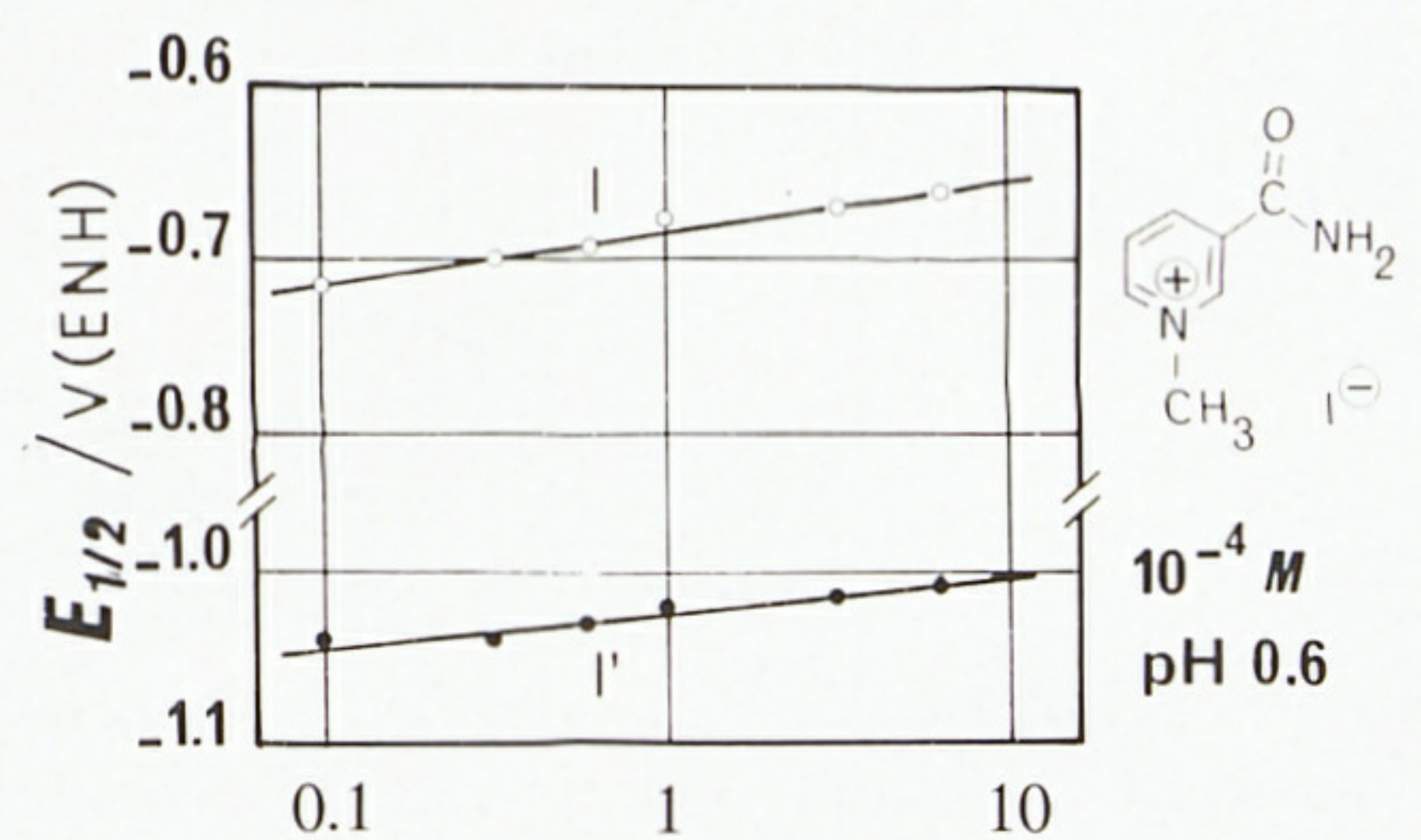

temps de goutte / s

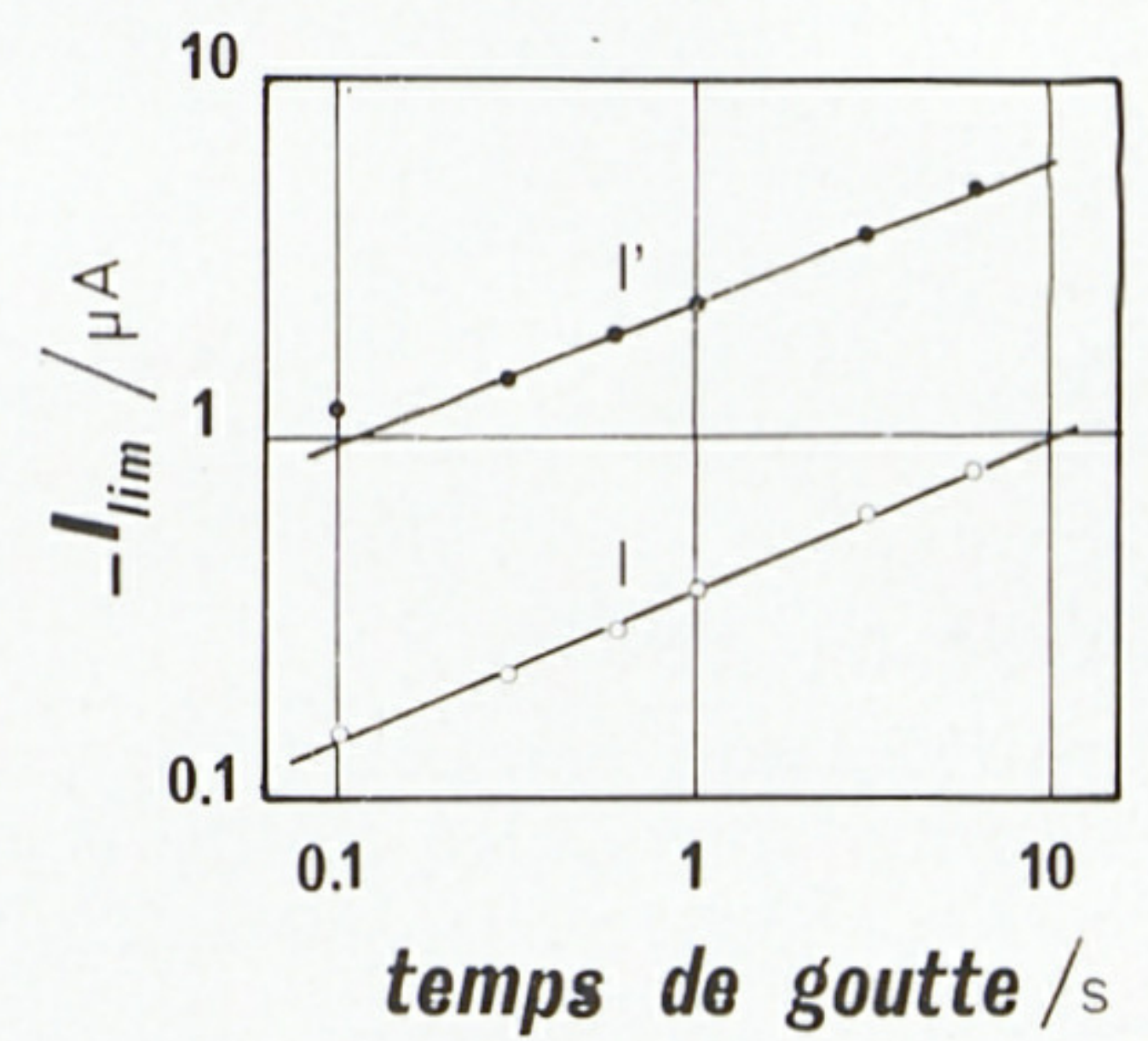

Fig. 7. Influence de la fréquence de chute des gouttes de mercure sur la réduction de l'iodure de $\mathrm{N}_{1}$-méthyl nicotinamide par polarographie à stillation commandée. Solution $10^{-4} M$ dans un tampon $0.5 M$ en acide chlorhydrique ( $\mathrm{pH}$ 0.58). La hauteur de la colonne de mercure est maintenue constante $(35 \mathrm{~cm})$ au-dessus du capillaire no. 10. Relation entre les potentiels de demi-vague et le logarithme du temps de goutte imposé.

Fig. 8. Influence de la fréquence de chute des gouttes de mercure sur la réduction de l'iodure de $\mathrm{N}_{1}$-méthyl nicotinamide par polarographieà stillation commandée. Conditions, voir Fig. 7. Relation entre le logarithme des hauteurs de vague et le logarithme du temps de goutte imposé.

observé par contre que les potentiels des différentes vagues augmentent tous linéairement avec le logarithme du temps de goutte imposé $\tau$. A pH 0.60 la première vague (I) se déplace très légèrement plus que la deuxième vague $\left(\mathrm{I}^{\prime}\right): \Delta E_{\frac{1}{2}} / \Delta \log \tau=+30 \pm 2$ et $+22 \pm 4 \mathrm{mV}$ respectivement pour les vagues I et I' (Fig. 7). A pH 9.57 la première vague II se déplace un peu moins que la deuxième vague III : $\Delta E_{\frac{1}{2}} / \Delta \log \tau=+18 \pm 2$ et $+29 \pm 2 \mathrm{mV}$ respectivement pour les vagues II et III (Fig. 9). Il est remarquable que l'influence de la fréquence de chute des gouttes de mercure sur les potentiels de demi-

J. Electroanal. Chem., 39 (1972) 
vague soit identique pour les deux vagues de réduction de l'iodure de $\mathrm{N}_{1}$-méthyl nicotinamide en milieu neutre ou légèrement basique (vagues II et III) et pour les deux vagues de réduction de la pyrimidine en milieu acide ${ }^{3}$.

Afin de déterminer la nature du phénomène qui limite la hauteur des différentes vagues de réduction de l'iodure de $\mathrm{N}_{1}$-méthyl nicotinamide, nous avons comparé l'influence du temps de goutte imposé $\tau$ tant sur la hauteur de ces vagues que sur la hauteur des vagues de réduction des cations $\mathrm{Tl}^{+}$et $\mathrm{Cd}^{2+}$. La réduction de $\mathrm{Tl}^{+}$et $\mathrm{Cd}^{2+}$ est en effet souvent utilisée comme exemple de réaction électrochimique réversible et rapide donnant des vagues dont la hauteur est limitée par la diffusion de réactif vers l'électrode ${ }^{6}$. Nous avons constaté que la hauteur des vagues de réduction de $\mathrm{Tl}^{+}$et $\mathrm{Cd}^{2+}$ en milieu acide varie linéairement avec une puissance $a$ du temps de goutte imposé $\tau$ :

$$
a=\Delta \log \left(-I_{\lim }\right) / \Delta \log \tau=0.25 \pm 0.07
$$

dans des conditions variées de concentration de réactif et de stillation du mercure. En milieu très acide ( $\mathrm{pH} 0.60)$ les deux vagues de réduction de l'iodure de $\mathrm{N}_{1}$-méthyl nicotinamide semblent avoir un comportement anormal vis-à-vis du temps de goutte imposé $\tau$ puisque $\Delta \log \left(-I_{\lim }\right) / \Delta \log \tau=0.44$ et 0.38 respectivement pour les vagues
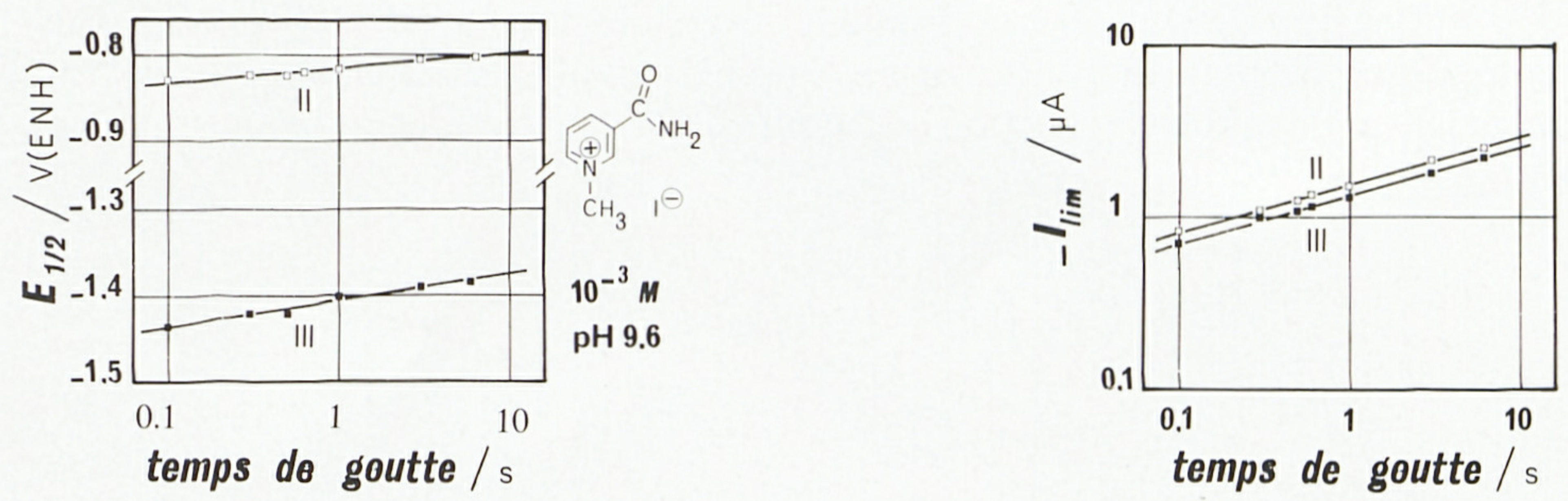

Fig. 9. Influence de la fréquence de chute des gouttes de mercure sur la réduction de l'iodure de $\mathrm{N}_{1}$-méthyl nicotinamide par polarographie à stillation commandée. Solution $10^{-3} M$ dans un tampon $0.5 M$ en acide borique et $0.5 \mathrm{M}$ en borate monopotassique ( $\mathrm{pH}$ 9.57). La hauteur de la colonne de mercure est maintenue constante $(35 \mathrm{~cm})$ au-dessus du capillaire no. 10. Relation entre les potentiels de demi-vague et le logarithme du temps de goutte imposé.

Fig. 10 Influence de la fréquence de chute des gouttes de mercure sur la réduction de l'iodure de $\mathrm{N}_{1}$-méthyl nicotinamide par polarographie à stillation commandée. Conditions, voir Fig. 9. Relation entre le logarithme des hauteurs de vague et le logarithme du temps de goutte imposé.

I et I' (Fig. 8). Au contraire, en milieu légèrement basique ( $\mathrm{pH} 9.57)$, les deux vagues ont des hauteurs qui semblent limitées par la diffusion de réactif vers l'électrode puisque $\Delta \log \left(-I_{\lim }\right) / \Delta \log \tau=0.25$ et 0.26 respectivement pour les vague II et III (Fig. 10$)$.

\subsection{Effet de la température de la solution}

La température $\theta$ de la solution n'a en général d'effet important sur le potentiel des vagues polarographiques que si celui-ci dépend essentiellement d'une vitesse de réaction chimique ou électrochimique ${ }^{7-11}$. Ceci n'est pas le cas des vagues I', II et III de réduction de l'iodure de $\mathrm{N}_{1}$-méthyl nicotinamide puisque leur potentiel de demivague ne varie pas entre 0 et $40^{\circ} \mathrm{C}$ (Figs. 11 et 13). Par contre, le potentiel de la vague I 
augmente légèrement avec la température $\theta$ en milieu très acide $(\mathrm{pH} 0.60)$ puisque $\Delta E_{\frac{1}{2}} / \Delta \theta=+1.0 \mathrm{mV}^{\circ} \mathrm{C}^{-1}$ (Fig. 11), ce qui reste compatible avec un transfert électronique rapide.

La valeur de l'influence de la température $\theta$ sur la hauteur des vagues polarographiques permet d'obtenir des indications sur le processus qui limite leur hauteur. D'après Kolthoff et Lingane ${ }^{12}$, Zuman ${ }^{6}$, Meites ${ }^{13}$ et Mairanovskii ${ }^{14}$ l'accroissement $^{\prime}$ relatif de la hauteur de vague $\left(\Delta I_{\mathrm{lim}} / I_{\mathrm{lim}}\right) / \Delta \theta$ vaut généralement $1.6 \pm 0.4 \%{ }^{\circ} \mathrm{C}^{-1}$ si ce

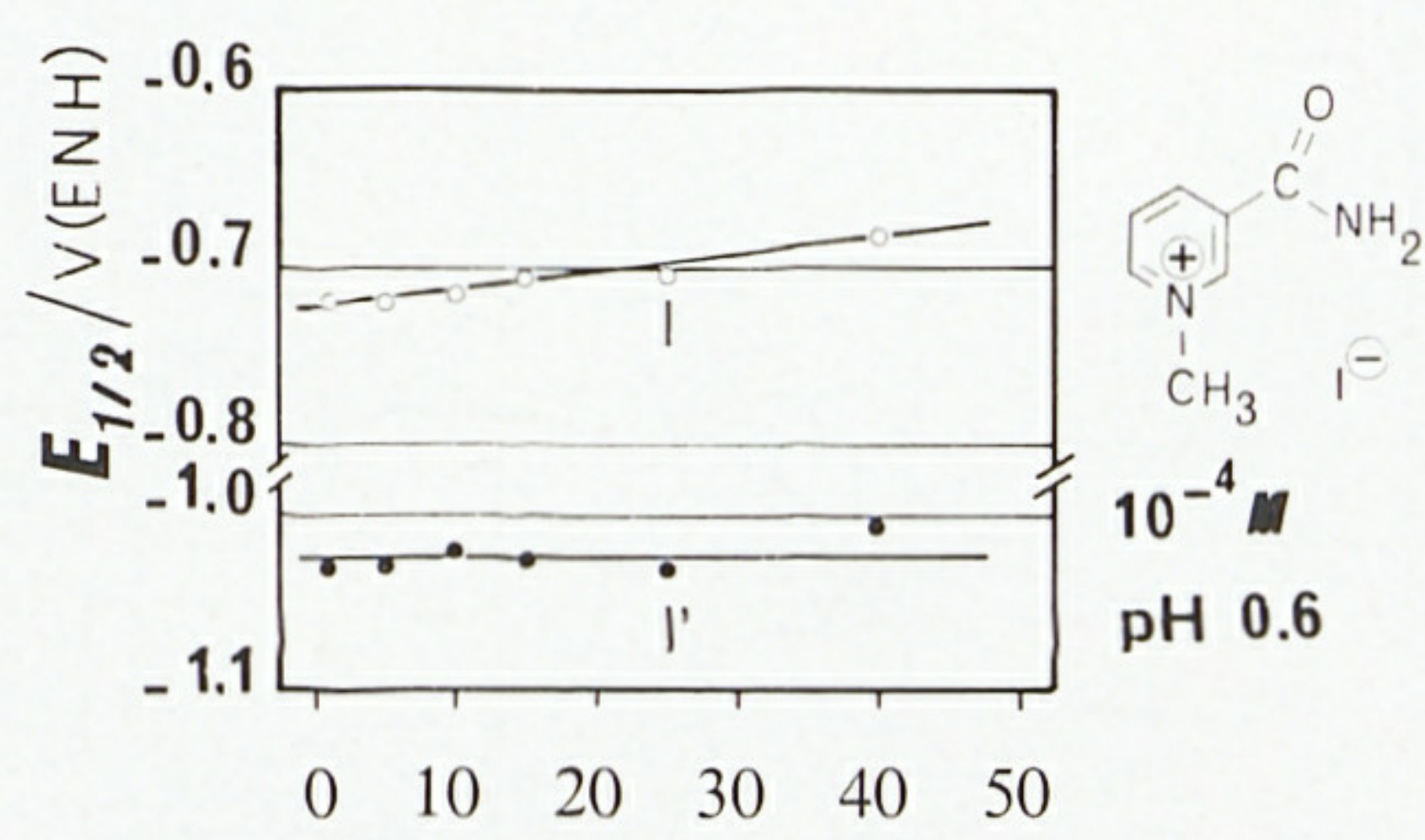

température $/{ }^{\circ} \mathrm{C}$

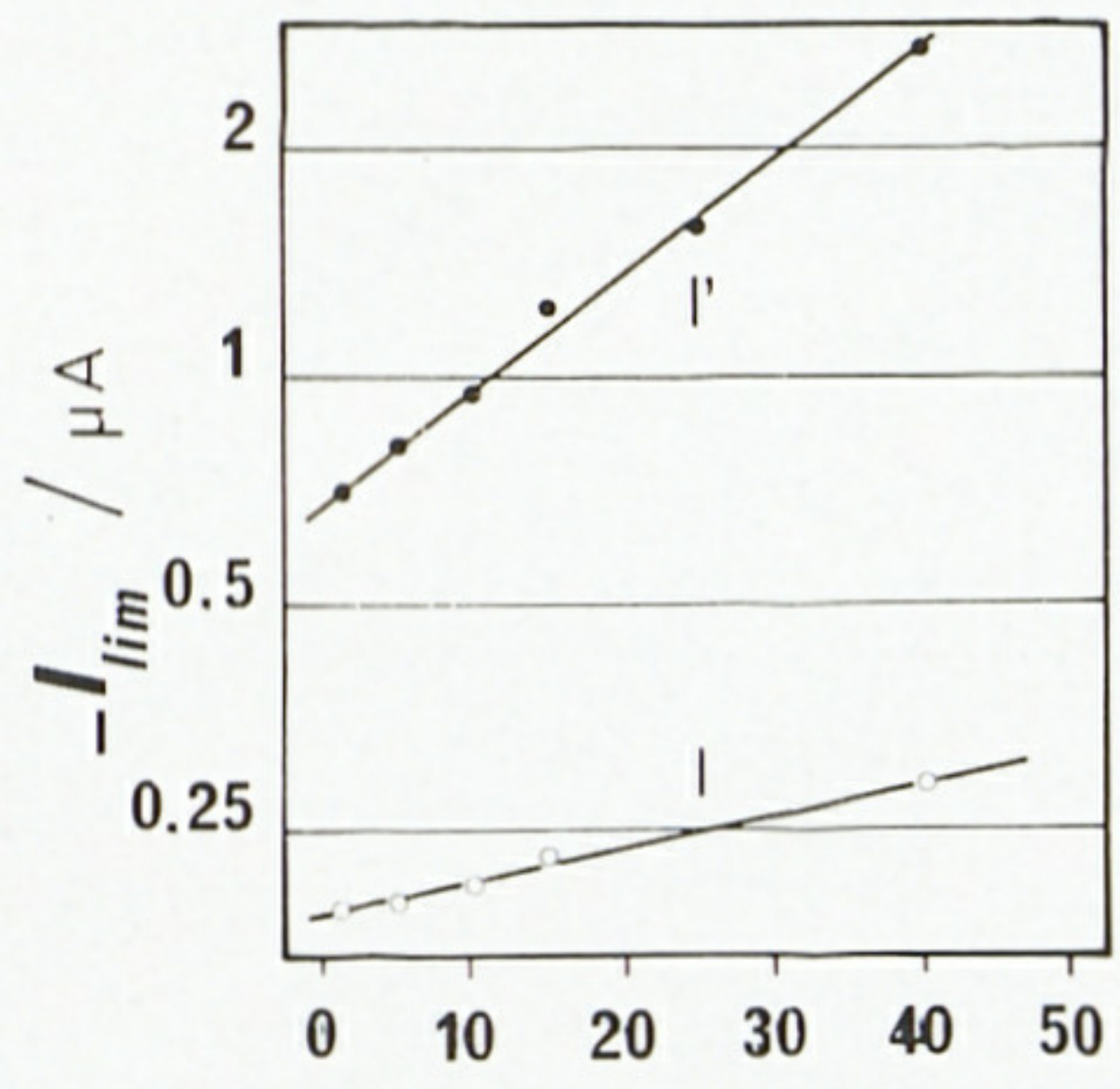

température $/{ }^{\circ} \mathrm{C}$

Fig. 11. Influence de la température sur la réduction de l'iodure de $\mathrm{N}_{1}$-méthyl nicotinamide par polarographie à stillation commandée. Solution $10^{-4} \mathrm{M}$ dans un tampon $0.5 \mathrm{M}$ en acide chlorhydrique ( $\mathrm{pH} 0.58$ ). Conditions polarographiques : capillaire no. $10,35.0 \mathrm{~cm}$ de mercure, temps de goutte imposé $0.50 \mathrm{~s}$. Relation entre les potentiels de demi-vague et la température.

Fig. 12. Influence de la température sur la réduction de l'iodure de $\mathrm{N}_{1}$-méthyl nicotinamide par polarographie à stillation commandée. Conditions, voir Fig. 11. Relation entre le logarithme des hauteurs de vague et la température.
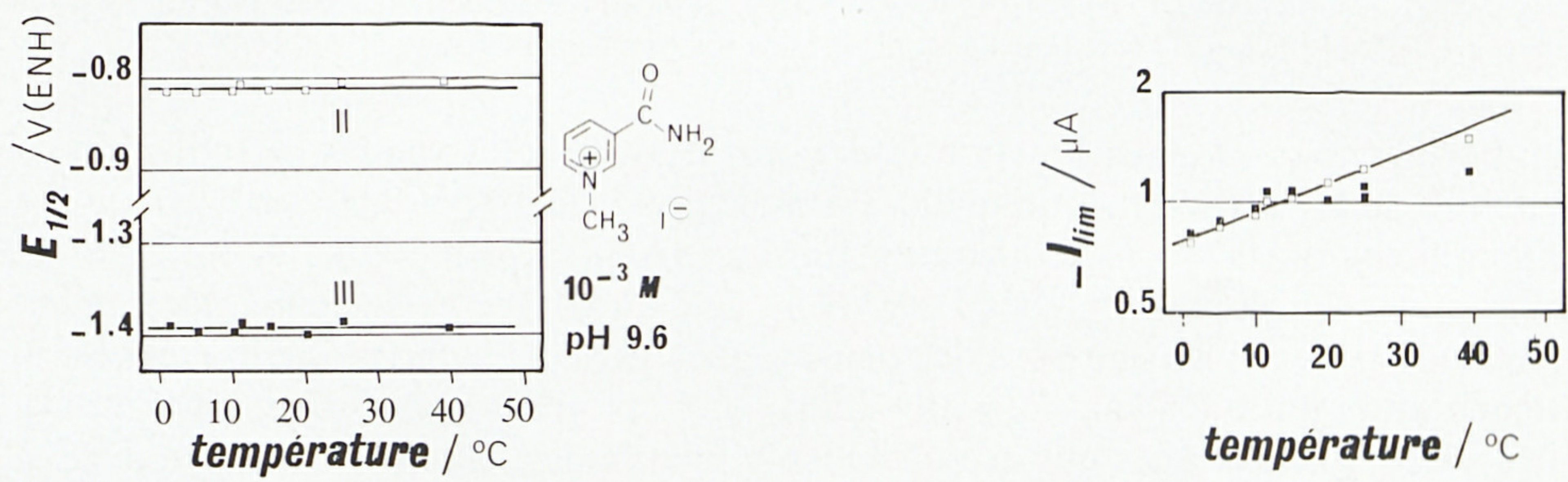

Fig. 13. Influence de la température sur la réduction de l'iodure de $\mathrm{N}_{1}$-méthyl nicotinamide par polarographie à stillation commandée. Solution $10^{-3} \mathrm{M}$ dans un tampon $0.5 \mathrm{M}$ en acide borique et $0.5 \mathrm{M}$ en borate monopotassique ( $\mathrm{pH}$ 9.57). Conditions polarographiques: capillaire no. $10,35.0 \mathrm{~cm}$ de mercure, temps de goutte imposé $0.50 \mathrm{~s}$. Relation entre les potentiels de demi-vague et la température.

Fig. 14. Influence de la température sur la réduction de l'iodure de $\mathrm{N}_{1}$-méthyl nicotinamide par polarographie à stillation commandée. Conditions, voir Fig. 13. Relation entre le logarithme des hauteurs de vague et la température.

processus est la diffusion de réactif à l'électrode et plus de $2.5 \%{ }^{\circ} \mathrm{C}^{-1}$ si c'est une cinétique de réaction. D’après Mairanovskii ${ }^{14}$ l'adsorbabilité des solutés diminuant généralement lorsque la température augmente, une valeur négative de $\Delta\left|I_{\text {lim }}\right| / \Delta \theta$ permettrait d'affirmer que le courant est limité par une adsorption du réactif sur l'électrode. Nous avons déterminé la valeur de cet accroissement relatif $\left(\Delta I_{\mathrm{lim}} / I_{\mathrm{lim}}\right) / \Delta \theta$ 

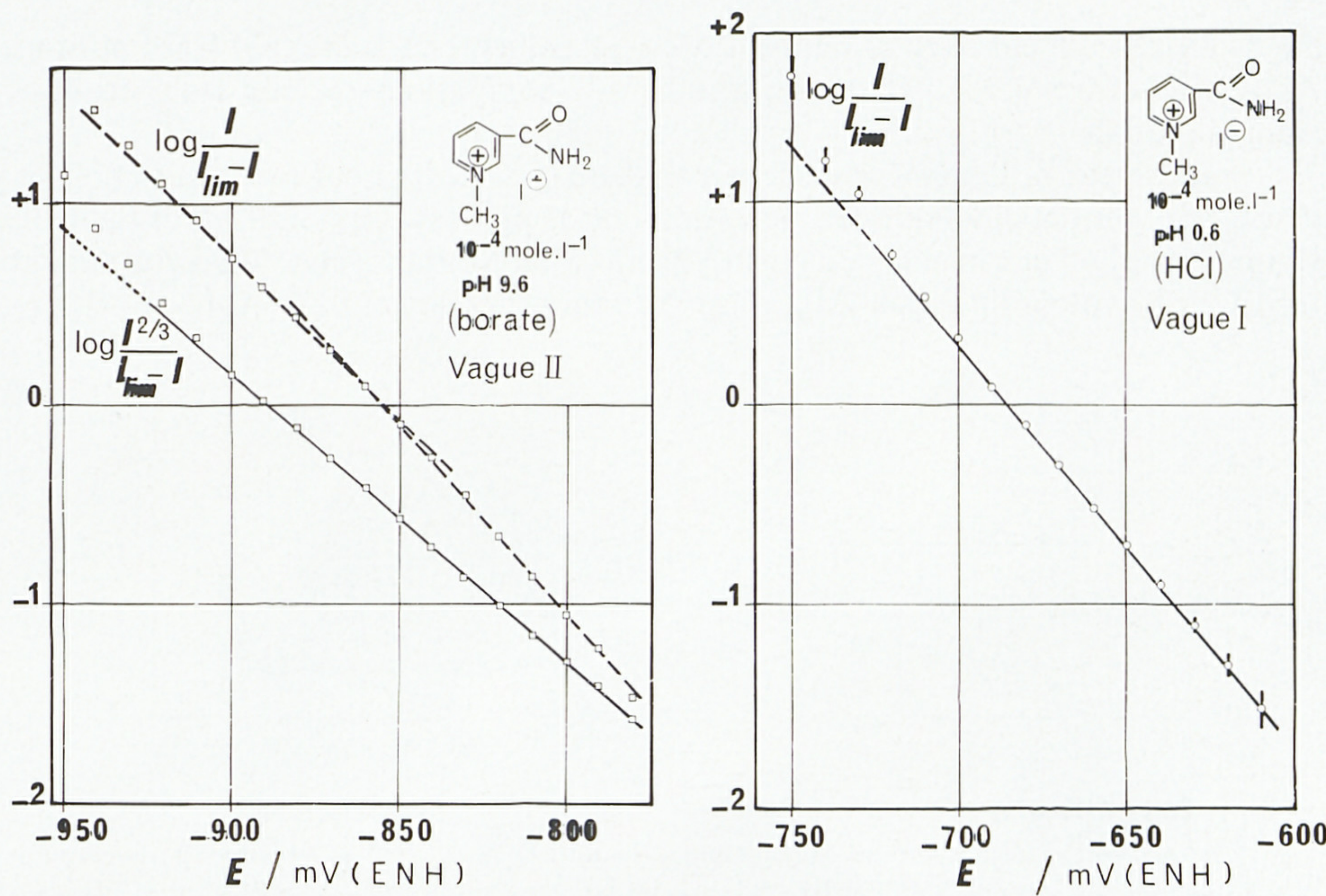

Fig. 15. Analyse logarithmique de la forme de la vague II de réduction de solutions $10^{-4} \mathrm{M}$ en iodure de $\mathrm{N}_{1}$-méthyl nicotinamide par polarographie à stillation commandée. Conditions polarographiques: capillaire no. $10,35.0 \mathrm{~cm}$ de mercure, temps de goutte imposé 0.50 s., $25.0 \pm 0.2^{\circ} \mathrm{C}$. Relation entre $(\square)$ $\log \left[I^{\frac{2}{3}} /\left(I_{\text {lim }}-I\right)\right]$ et le potentiel, $(---)\left[I /\left(I_{\text {lim }}-I\right)\right]$ et le potentiel à $\mathrm{pH} 9.57$ en tampon borate.

Fig. 16. Analyse logarithmique de la forme de la vague I de réduction de solutions $10^{-4} \mathrm{M}$ en iodure de $\mathrm{N}_{1}$-méthyl nicotinamide par polarographie à stillation commandée. Conditions, voir. Fig. 15. Relation entre $(-) \log \left[I /\left(I_{\mathrm{lim}}-I\right)\right]$ et le potentiel à pH 0.58 en tampon chlorhydrique.

en portant $\log \left(-I_{\text {lim }}\right)$ en fonction de $\theta$ pour les différentes vagues de réduction de l'iodure de $\mathrm{N}_{1}$-méthyl nicotinamide. En milieu très acide $(\mathrm{pH} 0.60)$ les deux vagues s'amplifient de façon fort différente puisque $\left(\Delta I_{\lim } / I_{\lim }\right) / \Delta \theta=0.92$ et $3.5 \%{ }^{\circ} \mathrm{C}^{-1}$ respectivement pour les vagues I et I' (Fig. 12). Au contraire, en milieu légèrement basique ( $\mathrm{pH}$ 9.57) les hauteurs des deux vagues varient de façon identique avec la température puisque $\left(\Delta I_{\lim } / I_{\lim }\right) / \Delta \theta=1.76 \%{ }^{\circ} \mathrm{C}^{-1}$ pour les vagues II et III (Fig. 14). Les valeurs numériques des accroissements relatifs des hauteurs de vague avec la température semblent donc exclure l'intervention d'une limitation par adsorption sur l'électrode ou par cinétique chimique ou électrochimique lente sauf dans ce dernier cas pour la vague I'.

\subsection{Analyse logarithmique de la forme des vagues}

En milieu très acide à $\mathrm{pH} 0.60$ la deuxième vague (vague $\mathrm{I}^{\prime}$ ) est déformée car proche du mur de réduction du solvant: l'analyse logarithmique de sa forme est donc sans grande signification. Par contre, dans le même milieu la première vague (vague I) présente la forme classique (Fig. 16): $\log \left[I /\left(I_{\lim }-I\right)\right]$ est proportionnel au potentiel avec une pente de $-1 / 50 \mathrm{mV}^{-1}$. Le rapport des hauteurs relatives des vagues I et II variant entre 2.6 et 3.2 , nous pouvons supposer l'existence probable, lors de la vague I, d'un transfert biélectronique lent (le produit $\alpha n$ est voisin de l'unité). 
L'effet de la concentration et du temps de goutte sur la position des deux vagues de réduction de l'iodure de $\mathrm{N}_{1}$-méthyl nicotinamide est semblable à celui observé pour la pyrimidine ${ }^{3}$, les sels de $\mathrm{N}_{1}$-alkyl pyridinium ${ }^{15,16}$, les aldéhydes et cétones aromatiques ${ }^{17-19}$ et les sels d'immonium ${ }^{20}$. Ce comportement a été généralement interprèté comme dû à une réaction cinétiquement du deuxième ordre entre les produits d'une réduction réversible ${ }^{22}$. A la suite des premiers travaux de Koutecky et Hanus ${ }^{21}$ de nombreux auteurs ont étudié la dimérisation électrochimique soit par polarographie $e^{15,19}$, soit par voltamétrie à balayage linéaire simple ${ }^{17}$ ou aller-retour $^{18,23}$ de potentiel, soit par chronoampérométrie simple ou aller-retour ${ }^{24}$. Récemment Savéant et coll. ${ }^{5,25,26}$ ont précisé, par le calcul, le comportement de vagues polarographiques ou de pics voltamétriques en tenant compte d'une part des valeurs de la concentration de réactif par rapport à la constante d'équilibre de dimérisation et d'autre part de la vitesse de diffusion des espèces vers l'électrode par rapport à la vitesse de dimérisation. Ces auteurs ont analysé les différents mécanismes de réaction possibles, c'est-à-dire:

- la dimérisation radicalaire:

$$
\begin{aligned}
& \mathrm{A}^{+}+e^{-} \rightleftarrows \mathrm{A}^{\cdot} \\
& 2 \mathrm{~A} \cdot \quad \rightleftarrows \mathrm{A}-\mathrm{A}
\end{aligned}
$$

- la dimérisation radical-ionique:

$$
\begin{aligned}
& \mathrm{A}^{+}+e^{-} \rightleftarrows \mathrm{A} \cdot \\
& \mathrm{A}^{\cdot}+\mathrm{A}^{+} \rightleftarrows \mathrm{A}-\mathrm{A}^{+} \\
& \mathrm{A}-\mathrm{A}^{+}+e^{-} \rightleftarrows \mathrm{A}-\mathrm{A} \\
& \mathrm{A}^{+}+e^{-} \rightleftarrows \mathrm{A}^{\cdot} \\
& \mathrm{A}^{\cdot}+e^{-} \rightleftarrows \mathrm{A}^{-} \\
& \mathrm{A}^{-}+\mathrm{A}^{+} \rightleftarrows \mathrm{A}-\mathrm{A}
\end{aligned}
$$$$
\text { - la dimérisation ionique : }
$$

Lorsque la vitesse de la réaction de dimérisation influe sur la réaction globale à l'électrode, c'est-à-dire lorsque la vitesse de cette dimérisation n'est ni trop lente, ni trop rapide comparée à la vitesse de diffusion et lorsque l'équilibre de dimérisation est favorable à la formation prépondérante de dimère $(K c \gg 1$ si $K$ est la constante de cet équilibre de dimérisation), les effets de la concentration $c$ et du temps de goutte $\tau$ sur $E_{\frac{1}{2}}$ permettent de distinguer entre ces trois types de mécanismes de dimérisation. En effet à $25^{\circ} \mathrm{C}$ :

$$
\begin{aligned}
& \Delta E_{\frac{1}{2}} / \Delta \log c=\Delta E_{\frac{1}{2}} / \Delta \log \tau=+19.7 \mathrm{mV} \text { si la dimérisation est radicalaire } \\
& \Delta E_{\frac{1}{2}} / \Delta \log c=\Delta E_{\frac{1}{2}} / \Delta \log \tau=+29.6 \mathrm{mV} \text { si la dimérisation est radical-ionique } \\
& \Delta E_{\frac{1}{2}} / \Delta \log c=\Delta E_{\frac{1}{2}} / \Delta \log \tau=+14.6 \mathrm{mV} \text { si la dimérisation est ionique }
\end{aligned}
$$

La valeur numérique que nous avons déterminée pour ces deux coefficients, respectivement $+19 \pm 1$ et $+18 \pm 2 \mathrm{mV}$ montre donc qu'en solution de concentration inférieure à $10^{-3} \mathrm{M}$ la vague II correspondrait à un transfert électronique suivi d'une dimérisation radicalaire à vitesse limitée. D'après Mairanovskii ${ }^{22}$ et Volke et al. ${ }^{16}$ cette vague II devrait avoir pour équation:

$$
E=E^{0}-(R T / n F) \log \left[I^{\frac{2}{3}} I_{\lim }^{\frac{1}{3}} /\left(I_{\lim }-I\right)\right]+(R T / n F) \log (c k \tau / 1.51)
$$

Dans la Fig. 15, tracée à partir de polarogrammes de la vague II obtenus à pH 9.57 et dans une solution $10^{-4} \mathrm{M}$ en réactif, nous avons porté conjointement en fonction du potentiel : $\log \left[I^{\frac{2}{3}} /\left(I_{\mathrm{lim}}-I\right)\right]$ et $\log \left[I /\left(I_{\mathrm{lim}}-I\right)\right]$. Il semble que la première expression (transfert électronique suivi d'une dimérisation) corresponde à une meilleure descrip- 
tion de la vague II dans ces conditions. En effet la quasi totalité des points obtenus par cette première expression sont alignés, alors que ceux obtenus par la deuxième (forme classique pour un transfert électronique rapide ou lent) sont alignés sur deux droites distinctes de part et d'autre du potentiel de demi-vague. Cependant les pentes des segments de droite sont tous assez différents de la valeur théorique $-1 / 58 \mathrm{mV}^{-1}$ attendue pour un transfert monoélectronique rapide. On relève en effet les valeurs de $-1 / 68 \mathrm{mV}^{-1}$ pour la première expression et de $-1 / 61$ et $-1 / 53 \mathrm{mV}^{-1}$ pour la deuxième expression. Enfin les résultats obtenus au même $\mathrm{pH}$ mais dans des solutions plus concentrées en iodure de $\mathrm{N}_{1}$-methyl nicotinamide sont qualitativement semblables, les pentes de ces segments de droite étant seulement légèrement modifiées.

Cet ensemble de résultats ne permettrait sans doute pas, s'il était pris isolément, de conclure sur l'intervention d'une réaction de dimérisation. Rapprochée des observations sur l'influence de la concentration de réactif et du temps de goutte imposé sur le potentiel de demi-vague, cette analyse logarithmique confirme l'existence d'un transfert monoélectronique suivi d'une dimérisation à vitesse limitée. L'écart entre les valeurs numériques réelles et théoriques de la pente de la transformée logarithmique de la vague II pourrait comme lors de la réduction des sels de $\mathrm{N}_{1}$-alkyl pyridinium ${ }^{22}$ être rattaché à une adsorption du dimère sur la surface de l'électrode. Cette dernière hypothèse est cependant en opposition avec les résultats de l'influence de la température sur la hauteur de la vague II (§ 3.3).

Dans le cas où une réaction de dimérisation a lieu concurrement à une deuxième étape de réduction, Mairanovskii ${ }^{22}$ à montré que la deuxième vague devrait avoir pour équation si elle est lente:

$$
E=\text { const. }-(2 R T / 3 \alpha n F) \log \left[I^{\frac{3}{2}} /\left(I_{\lim }-I\right)\right]
$$

Cette forme a été en effet observée dans le cas de la réduction du benzaldéhyde et de l'acétophénone ${ }^{22}$ et nous l'avons personnellement observée pour la vague II de la pyrimidine ${ }^{3}$. Par contre, dans des solutions tant $10^{-4} M$ que $10^{-2} M$ en iodure de $\mathrm{N}_{1}$-methyl nicotinamide à $\mathrm{pH} 9.57$ et 4.68 , la vague III ne présente pas plus cette forme que la forme classique.

\section{VOLTAMÉTRIE CYCLIQUE SUR DISQUE IMMOBILE DE CARBONE VITREUX: RÉDUCTION ET RÉOXYDATION}

De pH 6 à 10 l'iodure de $\mathrm{N}_{1}$-méthyl nicotinamide en solution aqueuse $10^{-3} \mathrm{M}$ donne un seul pic de réduction en voltamétrie à balayage rapide sur électrode immobile à disque de carbone vitreux. Comme l'illustre la Fig. 17 le potentiel de ce pic, mesuré pour une vitesse de balayage du potentiel de $0.50 \mathrm{~V} \mathrm{~s}^{-1}$, ne dépend pas du $\mathrm{pH}$ :

$$
\text { pic II } \quad E_{\mathrm{p}}=-850 \pm 20 \mathrm{mV} / \mathrm{ENH}
$$

Le potentiel de ce pic est légèrement plus négatif $(20-30 \mathrm{mV})$ que $E_{\frac{1}{2}}$ de la première vague polarographique (vague II) relevée dans les mêmes conditions de concentration et de milieu. Ce résultat est en accord avec l'hypothèse d'un transfert réversible d'un électron; en effet dans ce cas on doit avoir ${ }^{27}$ :

$$
E_{\mathrm{p}}=E_{\frac{1}{2}}-1.109 R T / n F
$$

soit à $25^{\circ} \mathrm{C}$ :

$$
E_{\mathrm{p}}=E_{\frac{1}{2}}-0.0285 / n \mathrm{~V}
$$




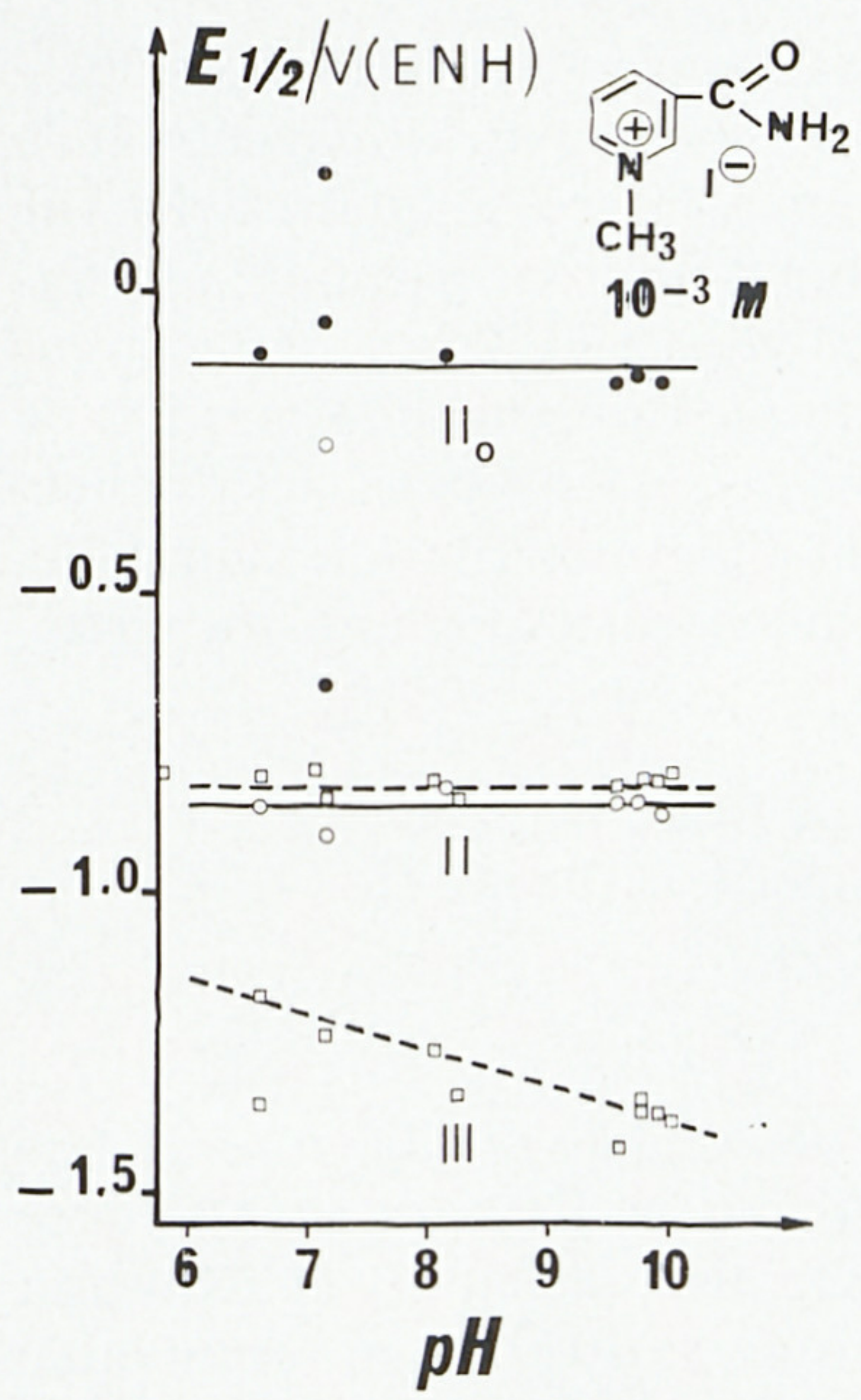

Fig. 17. Réduction de solns. aqueuses $10^{-3} \mathrm{M}$ en iodure de $\mathrm{N}_{1}$-méthyl nicotinamide par polarographie à stillation commandée $(\square)$ et par voltamétrie à balayage linéaire de potentiel $\left(0.5 \mathrm{~V} \mathrm{~s}^{-1}\right)$ sur électrode immobile à disque de carbone vitreux polie mécaniquement avant chaque tracé $(\bigcirc)$. Pic de réoxydation $(\boldsymbol{O})$ observé en voltamétrie cyclique sur électrode immobile à disque de carbone vitreux si le balayage linéaire du potentiel $\left(0.5 \mathrm{~V} \mathrm{~s}^{-1}\right)$ est inversé à environ $-1.3 \mathrm{~V} / \mathrm{ENH}$. Influence du $\mathrm{pH}$ de la soln. sur les potentiels de demi-vague (polarographie) et sur les potentiels de pic (voltamétrie).

Il est également clair d'après la proximité de ces potentiels obtenus sur électrode de mercure ou de carbone vitreux que le mécanisme de cette première étape de réduction ne fait pas intervenir le matériau de l'électrode indicatrice.

Lorsque le sens du balayage des potentiels est inversé vers $-1.3 \mathrm{~V} / \mathrm{ENH}$, le balayage retour donne un pic d'oxydation $\mathrm{II}_{0}$ quelle que soit la vitesse de ce balayage retour (Fig. 17). Ce pic anodique $\mathrm{II}_{0}$ disparaît lorsque le potentiel d'inversion de sens du balayage est supérieur à $-0.85 \mathrm{~V} / \mathrm{ENH}$. Il correspond donc à la réoxydation du produit de réduction du pic II. A l'exception des valeurs obtenues dans le tampon imidazole ( $\mathrm{pH}$ 7.15), pour lequel il semble qu'il apparaisse plusieurs pics de réduction et de réoxydation, le potentiel du pic $\mathrm{II}_{0}$ anodique ne dépend pas du $\mathrm{pH}$ de la solution, ni de la nature du tampon lorsque le $\mathrm{pH}$ est compris entre 6 et 10. Lorsque les balayages en potentiel sont effectués, à l'aller comme au retour, à $0.50 \mathrm{~V} \mathrm{~s}^{-1}$, le pic $\mathrm{II}_{0}$ a pour potentiel:

$$
\left(E_{\mathrm{p}}\right)_{\mathrm{II}_{0}}=-110 \pm 20 \mathrm{mV} / \mathrm{ENH}
$$

Sa hauteur et sa surface sont souvent voisines de celles du pic cathodique. A l'exception encore des résultats obtenus avec le tampon imidazole $(\mathrm{pH} 7.15)$, une électrolyse pendant $60 \mathrm{~s}$ à $-1.3 \mathrm{~V}$ (potentiel inférieur de $450 \mathrm{mV}$ à celui du pic de réduction) ne modifie pas considérablement la forme des courbes intensité-potentiel ni la taille du pic de réoxydation.

L'absence d'effet du pH de la solution sur les potentiels des pics de réduction 
et de réoxydation montre que les mécanismes de ces deux réactions ne font pas intervenir d'échange de protons. La similitude entre les surfaces des pics de réduction et de réoxydation laisse penser que le produit de réduction est stable et qu'il est réoxydé par un mécanisme faisant intervenir le même nombre d'électrons par motif nicotinamide que la réduction. Pour la vitesse de balayage utilisée $\left(0.50 \mathrm{~V} \mathrm{~s}^{-1}\right)$ aucun pic anodique n'a été observé au voisinage immédiat du pic cathodique: le produit de réduction doit donc donner lieu à une réaction chimique assez rapide-les résultats polarographiques ont montré ( $c f$. $§ 1.3$ ) qu'il s'agirait d'une dimérisation radicalaire. Le pic anodique, situé à $740 \pm 40 \mathrm{mV}$ du pic cathodique, correspondrait par conséquent à une oxydation du dimère $\mathrm{A}-\mathrm{A}$.

\section{DISCUSSION ET INTERPRÉTATION DES RÉSULTATS DES MESURES ÉLECTROCHIMIQUES SUR LES SOLUTIONS AQUEUSES DE L'IODURE DE $\mathrm{N}_{1}$-MÉTHYL NICOTINAMIDE}

\subsection{Comparaison avec les données antérieures}

Les données électrochimiques que nous avons réunies sur l'iodure de $\mathrm{N}_{1}$ méthyl nicotinamide en solution aqueuse confirment, en les étendant et en les précisant, les données antérieures. Ces dernières concernent seulement la réduction polarographique de solutions de concentration voisine de $10^{-3} \mathrm{M}$ en réactif et de $\mathrm{pH}$ généralement compris entre 5 et 10 . Nous avons par conséquent comparé ces données à celles que nous avons obtenues par polarographie à stillation commandée de solutions $10^{-3} \mathrm{M}$ en réactif (Fig. 18). Les quelques valeurs de potentiel de demivague relevées par Leach et al. ${ }^{30}$ sont supérieures de 100-140 mV pour la vague II et

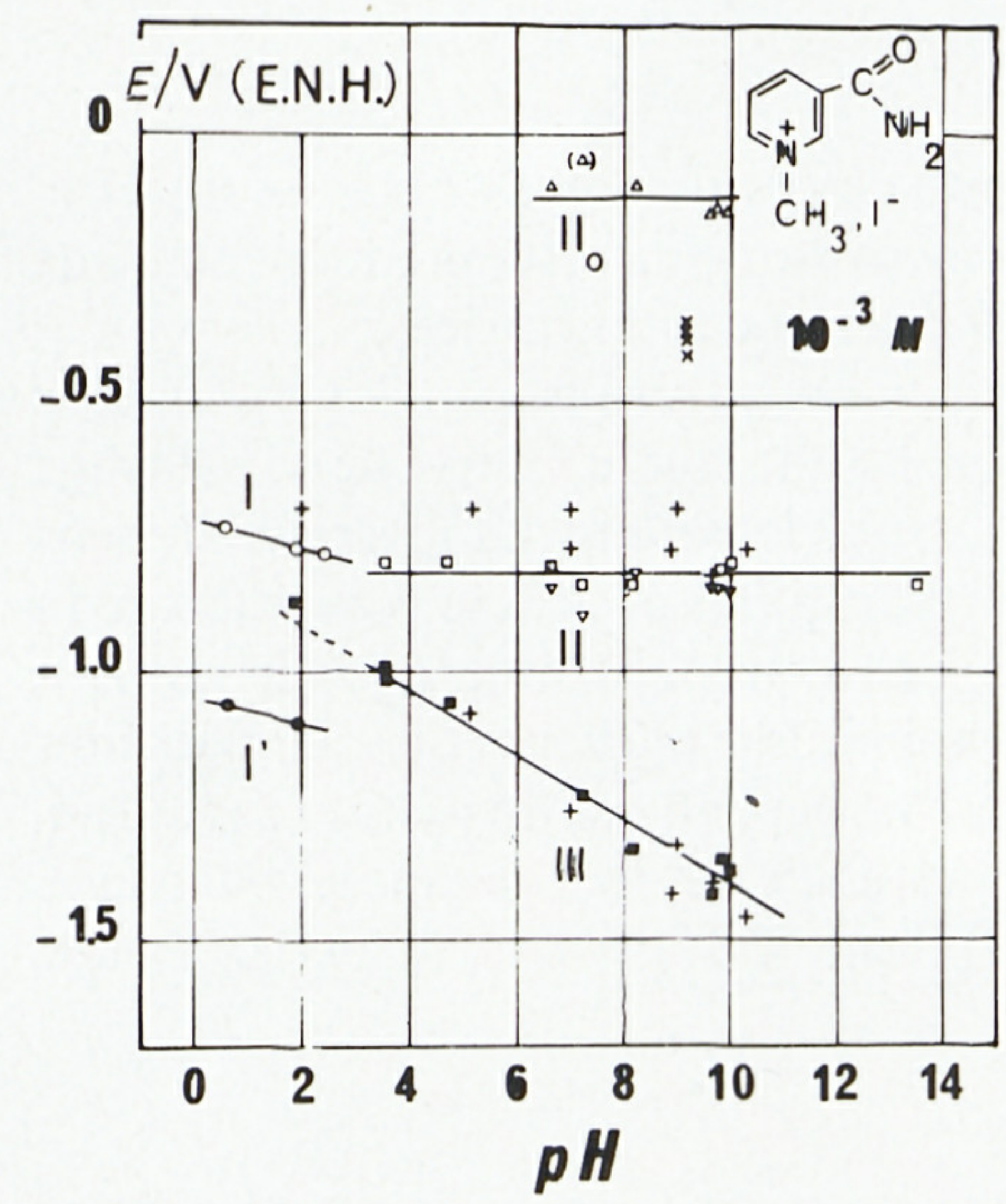

Fig. 18. Electrochimie de solns. aqueuses $10^{-3} M$ en iodure de $\mathrm{N}_{1}$-méthyl nicotinamide. Résultats antérieurs relatifs à la réduction polarographique de l'iodure de $\mathrm{N}_{1}$-méthyl nicotinamide $(+)^{30-32}$ et à la potentiométrie de mélanges d'iodure de $\mathrm{N}_{1}$-méthyl nicotinamide et de 1,4-dihydro- $\mathrm{N}_{1}$-méthyl nicotinamide en quantité équivalente et en présence de médiateur redox $(x)^{32}$. Résultats obtenus avec l'iodure de N-méthyl nicotinamide par réduction par polarographie à stillation commandée (vagues $\mathrm{I}(\bigcirc), \mathrm{I}^{\prime}(\bigcirc), \operatorname{II}(\square), \operatorname{III}(\mathbf{⿴ 囗 十})$ ) et par voltamétrie cyclique sur électrode immobile à disque de carbone vitreux tant en réduction $(\nabla)$ qu'en réoxydation $(\triangle)$ à $0.5 \mathrm{~V} \mathrm{~s}^{-1}$. 
égales pour la vague III aux valeurs que nous avons relevées à mêmes $\mathrm{pH}$. Les valeurs mesurées à pH 9.65 par Ciusa et al. ${ }^{29}$ sont en excellent accord avec les nôtres. Enfin, Nakaya ${ }^{28}$ a déterminé des potentiels de demi-vague qui comparés à ceux que nous avons mesurés aux mêmes $\mathrm{pH}$ sont supérieurs d'environ $40 \mathrm{mV}$ pour la vague II et inférieurs d'environ $50 \mathrm{mV}$ pour la vague III.

Les données électrochimiques que nous avons réunies apportent d'autre part trois types de résultats nouveaux que nous avons condensés sur la Fig. 18:

- La réduction polarographique fait apparaître à $\mathrm{pH}$ inférieur à environ 3 une vague I dont la hauteur est voisine de la somme des hauteurs des vagues II et III en milieu neutre.

- La réduction sur disque de carbone vitreux immobile fournit dans des solutions de $\mathrm{pH}$ compris entre 6.5 et 10 un pic voltamétrique II dont la position coïncide avec celle de la première vague polarographique dans ce domaine de $\mathrm{pH}$ (vague II).

— La réoxydation du produit de réaction formé sur le pic précédent fournit dans les mêmes conditions un pic voltamétrique $\mathrm{II}_{0}$ situé, quelque soit le $\mathrm{pH}$, à un potentiel supérieur de $740 \mathrm{mV}$ environ au potentiel du pic précédent.

Enfin, au cours de cette étude électrochimique de l'iodure de $\mathrm{N}_{1}$-méthyl nicotinamide nous avons clairement mis en évidence l'influence de paramètres expérimentaux qui avaient été incomplètement étudiés (concentration de réactif) ou totalement oubliés (nature du tampon, temps de goutte, température). Nous avons relevé l'influence de ces facteurs tant sur la hauteur des vagues polarographiques, ce qui dans quelques cas avait été partiellement réalisé, que sur la valeur de leur potentiel de demi-vague. C'est grâce à la combinaison de ces résultats expérimentaux que nous pouvons envisager de reprendre et de préciser les hypothèses relatives aux mécanismes de ces processus et de démontrer en particulier l'existence d'une dimérisation radicalaire directement à partir des relevés polarographiques. On sait en effet qu'il y a souvent des difficultés à passer des résultats obtenus par macroélectrolyse à potentiel contrôlé à des mécanismes de réactions électrochimiques sur microélectrode.

\subsection{Interprétation des résultats en termes de mécanismes de réactions}

(a) Lorsque le $\mathrm{pH}$ de la solution est très acide, la première étape de réduction de l'iodure de $\mathrm{N}_{1}$-méthyl nicotinamide (vague I) est d'une grande complexité et les résultats obtenus ne permettent pas encore d'interpréter avec certitude toutes ses caractéristiques.

Les potentiels de demi-vague que nous avons relevés dans des tampons de nature et de $\mathrm{pH}$ différents diminuent de façon significative lorsque le $\mathrm{pH}$ augmente. Ceci montre que la réduction fait intervenir un échange de protons avant ou peu après le transfert d'électrons. Cette variation ne semble cependant qu'approximativement linéaire $\left(\Delta E_{\frac{1}{2}} / \Delta \mathrm{pH}=80 \pm 20 \mathrm{mV}\right)$ et nous ne pouvons à partir des valeurs obtenues définir avec précision le rapport du nombre de protons et d'électrons échangés. L'influence de la concentration de réactif et de la température de la solution sur la position et la hauteur de la vague I montre que le facteur limitant le courant est la diffusion du réactif vers l'électrode. L'analyse de la forme de cette vague montre que l'étape primaire correspond à un transfert électronique rapide ou lent. La rapprochement de la valeur numérique de la pente de la vague $\mathrm{I}$, repérée par $1 /\left|E_{\frac{3}{4}}-E_{\frac{1}{4}}\right|$, soit $1 /(50 \pm 5) \mathrm{mV}^{-1}$, et du rapport des hauteurs des vagues I et II, soit 2.6-3.2, conduit 
à l'hypothèse plausible d'un transfert biélectronique lent. Cependant la vague I correspond certainement à un ensemble complexe de réactions chimiques et électrochimiques puisque le potentiel de demi-vague dépend de façon significative du temps de goutte imposé:

$\Delta E_{\frac{1}{2}} / \Delta \log \tau=+30 \pm 2 \mathrm{mV}$

(b) Lorsque le $\mathrm{pH}$ est compris entre 3 et 10 la première étape de réduction de l'iodure de $\mathrm{N}_{1}$-méthyl nicotinamide, tant sur goutte de mercure à stillation commandée que sur disque de carbone vitreux, est celle pour laquelle les résultats sont les plus nombreux et les plus concordants. L'absence d'effet du $\mathrm{pH}$ sur la position et sur la hauteur de cette vague II montre que la réduction ne fait intervenir d'échange de proton ni avant, ni après le transfert des électrons. L'analyse logarithmique de la forme de cette vague II, en accord avec les coulométries effectuées sur son palier de diffusion $^{31}$ permet de supposer qu'un seul électron est alors fixé sur une molécule de réactif. La concentration $c$ de réactif et la période $\tau$ de stillation des gouttes de mercure ayant toutes deux une influence sur le potentiel de demi-vague, le transfert électronique est suivi d'une réaction chimique d'ordre différent de un. Les valeurs relevées tant pour $\Delta E_{\frac{1}{2}} / \Delta \log c$ que pour $\Delta E_{\frac{1}{2}} / \Delta \log \tau$, respectivement $19 \pm 1$ et $18 \pm 2$ $\mathrm{mV}$ dans des solutions $10^{-4} M$ en réactif, sont en excellent accord avec la valeur théorique de ces deux coefficients $(19.7 \mathrm{mV})$ correspondant à une réaction consécutive selon laquelle le radical $\mathrm{A} \cdot$, formé à l'électrode, se dimérise assez rapidement:

$$
2 \mathrm{~A} \cdot \rightarrow \mathrm{A}-\mathrm{A}
$$

L'analyse logarithmique de la forme de cette vague II dans des solutions $10^{-4} \mathrm{M}$ en réactif conduit au même mécanisme. Notons que la vitesse de cette dimérisation n'est pas assez élevée pour que le mécanisme de la réduction à l'électrode soit:

$$
2 \mathrm{~A}^{+}+2 e^{-} \rightarrow \mathrm{A}-\mathrm{A}
$$

En effet si c'était le cas $E_{\frac{1}{2}}$ augmenterait de $60 \mathrm{mV}$ par décade de concentration et ne dépendrait pas du temps de goutte imposé. Le potentiel de la vague II correspond donc au seul transfert électronique:

$$
\mathrm{A}^{+}+e^{-} \rightarrow \mathrm{A}^{\cdot}
$$

la réaction de dimérisation n'intervenant sur la forme et la position de cette vague que par modification de la concentration de radical libre A· au voisinage de l'électrode. Ceci est en accord avec la réversibilité du transfert électronique mise en évidence par voltamétrie cyclique à balayage très rapide de potentiel $\left(20-50 \mathrm{~V} \mathrm{~s}^{-1}\right)$ avec des solutions aqueuses de chlorure de $\mathrm{N}_{1}$-méthyl nicotinamide ${ }^{32}$ : Underwood et al. ont observé dans ces conditions, au cours du balayage retour, un pic anodique décalé de 10-50 $\mathrm{mV}$ seulement du pic cathodique.

Finalement cette première étape de réduction en solution aqueuse diluée de $\mathrm{pH}$ compris entre 3 et 10 correspond à

$$
\begin{aligned}
\mathrm{A}^{+}+e^{-} \rightleftharpoons \mathrm{A} \cdot \quad & E_{\mathrm{II}}^{0}=\left(E_{\frac{1}{2}}\right)_{\mathrm{II}}-(R T / 3 n F) \log (c k \tau / 2.95) \\
& E_{\mathrm{II}}^{0}=-0.83-0.020 \log \left(10^{-4} \times 10^{7} \times 0.5 / 3\right) \\
& E_{\mathrm{II}}^{0}=-0.87 \mathrm{~V} / \mathrm{ENH}
\end{aligned}
$$


(si l'on prend pour estimation de la constante de vitesse de dimérisation celle obtenue par Land et Swallow ${ }^{34}$ par radiolyse pulsée) suivie de

$$
2 \mathrm{~A} \cdot \rightleftharpoons \mathrm{A}-\mathrm{A} \quad K_{\mathrm{II}}=[\mathrm{A}-\mathrm{A}] /[\mathrm{A} \cdot]^{2}
$$

Lorsque pour le même domaine de $\mathrm{pH}$, la concentration de l'iodure de $\mathrm{N}_{1}$-méthyl nicotinamide est supérieure à environ $10^{-3} M, E_{\frac{1}{2}}$ n'augmente plus avec cette concentration mais reste constant ou même diminue très faiblement. Il se pourrait alors que, préalablement à sa réduction et lorsque sa concentration est supérieure à environ $1 \mathrm{mM}$, ce réactif $\mathrm{A}^{+}$se dimérise en $\mathrm{A}^{+}-\mathrm{A}^{+}$.

(c) Toujours pour les $\mathrm{pH}$ compris entre 3 et 10, la deuxième étape de réduction de l'iodure de $\mathrm{N}_{1}$-méthyl nicotinamide est située à un potentiel si négatif qu'elle ne peut être observée que sur électrode de mercure. Même lorsque cette vague III n'est pas déformée et présente une hauteur sensiblement égale à celle de la vague II $(\mathrm{pH}$ compris entre 8 et 10), il n'est pas possible de définir complètement et avec sûreté le mécanisme de cette deuxième étape de réduction tant les phénomènes observés sont complexes. L'égalité des hauteurs des vagues II et III conduit à penser que lors de cette deuxième étape de réduction un seul électron est à nouveau fixé sur une moléle de réactif. La variation de $E_{\frac{1}{2}}$ avec le $\mathrm{pH}$, pour une même concentration de réactif, est égale à $60 \mathrm{mV}$ par unité de $\mathrm{pH}$. Il est donc probable que le nombre de protons fixé par molécule de réactif soit égal au nombre d'électrons donc à un. Les électrolyses effectuées au potentiel de cette vague III ayant fourni des rendements élevés en un dérivé dihydro, il est permis de penser que le produit de cette deuxième étape de réduction de $\mathrm{A}^{+}$est finalement $\mathrm{AH}$. Plusieurs mécanismes de réaction sont alors possibles :

$$
\mathrm{A} \cdot+e^{-}+\mathrm{H}^{+} \rightarrow \mathrm{AH} \text { concurrente avec } 2 \mathrm{~A} \cdot \rightarrow \mathrm{A}-\mathrm{A}
$$

ou bien

$$
\mathrm{A}-\mathrm{A}+2 e^{-}+2 \mathrm{H}^{+} \rightarrow 2 \mathrm{AH}
$$

ou bien

$$
\mathrm{A}-\mathrm{A}+2 e^{-}+2 \mathrm{H}^{+} \rightarrow \mathrm{AH}-\mathrm{AH} \rightarrow 2 \mathrm{AH}
$$

L'importance des effets sur $E_{\frac{1}{2}}$ de la concentration totale de réactif $c\left(\Delta E_{\frac{1}{2}} / \Delta\right.$ $\log c=-43 \pm 2 \mathrm{mV})$ et du temps de goutte imposé $\tau\left(\Delta E_{\frac{1}{2}} / \Delta \log \tau=+29 \pm 2 \mathrm{mV}\right)$ montre que la réaction électrochimique est complexe et fait certainement intervenir une ou plusieurs réactions chimiques antérieures, concurrentes ou postérieures au transfert des électrons et protons. L'absence d'effet de la température sur le potentiel de demi-vague montre que celui-ci ne dépend pas essentiellement d'une cinétique de réaction. La comparaison de l'influence du temps de goutte imposé sur la hauteur de cette vague et sur la hauteur des vagues de réduction de $\mathrm{Tl}^{+}$et $\mathrm{Cd}^{2+}$ conduit au même résultat : le phénomène qui limite le courant de la vague III est bien la diffusion de réactif vers l'électrode. Seule l'étude de la réoxydation du ou des produits de réduction de cette vague III, soit par voltamétrie cyclique à balayage rapide de potentiel, soit par voltamétrie sur électrode disque-anneau, permettra sans doute d'élucider le mécanisme de cette deuxième étape de la réduction de l'iodure de $\mathrm{N}_{1}$-méthyl nicotinamide en milieu neutre ou basique.

(d) La voltamétrie cyclique à balayage modérément rapide $\left(0.5 \mathrm{~V} \mathrm{~s}^{-1}\right)$ sur 
disque immobile de carbone vitreux nous a montré, qu'entre $\mathrm{pH} 6.5$ et 10, la position du pic de réoxydation du produit de la première étape de réduction ne dépend pas du $\mathrm{pH}$. Cette étape de réoxydation ne contient donc pas d'échange de proton. La surface du pic anodique au cours du balayage retour étant assimilable à celle du pic cathodique, nous pouvons supposer que le nombre d'électrons échangés pour cette réoxydation est le même qu'à la réduction, c'est-à-dire un électron par motif $\mathrm{N}_{1}$-méthyl nicotinamide. Comme le produit de la première étape de réduction est en milieu neutre le dimère $\mathrm{A}-\mathrm{A}$, cette étape de réoxydation pourrait correspondre à l'un des mécanismes de réaction suivants

$$
\mathrm{A}-\mathrm{A} \rightarrow 2 \mathrm{~A}^{+}+2 e^{-}
$$

ou

$$
\mathrm{A}-\mathrm{A} \rightarrow \mathrm{A}^{+}-\mathrm{A}^{+}+2 e^{-} \rightleftarrows 2 \mathrm{~A}^{+}
$$

ou encore

$$
\mathrm{A}-\mathrm{A} \rightleftarrows 2 \mathrm{~A} \cdot \rightarrow 2 \mathrm{~A}^{+}+2 e^{-}
$$

Seule une étude plus complète, en particulier de l'influence de la concentration de réactif et de la vitesse de balayage des potentiels, permettrait de choisir entre ces hypothèses.

En conclusion, la détermination des étapes réactionnelles correspondant aux potentiels de transition redox de l'iodure de $\mathrm{N}_{1}$-méthyl nicotinamide ou de ses dérivés réduits, relevés par polarographie, voltamétrie ou potentiométrie, n'en est encore sur certains points qu'au stade des hypothèses. Il existe cependant un cas où le mécanisme peut sembler convenablement établi: il s'agit de la première étape de réduction de solutions diluées de l'iodure de $\mathrm{N}_{1}$-méthyl nicotinamide de $\mathrm{pH}$ compris entre 3 et 10 .

\subsection{Energétique des mécanismes de réactions proposés}

Après avoir essayé d'établir une correspondance entre potentiel et mécanisme de réaction séparément pour chaque phénomène observé, il est ensuite nécessaire d'effectuer un contrôle interne sur l'ensemble de ces hypothèses en appliquant les lois d'additivité Nous considérons comme correctement établi le potentiel normal $E^{0}$ du couple $\mathrm{A}^{+} / \mathrm{AH}$ relevé par potentiométrie d'un mélange d'iodure de $\mathrm{N}_{1}$-méthyl nicotinamide et de 1,4-dihydro- $\mathrm{N}_{1}$-méthyl nicotinamide en présence de médiateur $\operatorname{redox}^{30}$ :

$$
\mathrm{A}^{+}+2 e^{-}+\mathrm{H}^{+} \rightleftarrows \mathrm{AH} \quad E^{0}=-0.360 \pm 0.005 \mathrm{~V} / \mathrm{ENH} \text { à } \mathrm{pH} 9.1
$$

La deuxième réaction que nous pouvons considérer comme définie quant à son potentiel normal est celle qui correspond à la formation du radical libre $\mathrm{A}$. (vague ou pic II):

$$
\mathrm{A}^{+}+e^{-} \rightleftharpoons \mathrm{A} \cdot \quad E_{\mathrm{II}}^{0}=-0.87 \pm 0.02 \mathrm{~V} / \mathrm{ENH} \text { entre } \mathrm{pH} 3 \text { et } 10
$$

Ainsi que nous l'avons montré précédemment ${ }^{35}$, à partir des données électrochimiques publiées sur le NAD, un des mécanismes proposés pour l'interprétation de la vague III doit être exclu. En effet

$$
\mathrm{A} \cdot+e^{-}+\mathrm{H}^{+} \rightarrow \mathrm{AH}
$$


ne peut s'effectuer à $E_{\mathrm{III}}^{0} \simeq-1.35 \mathrm{~V} / \mathrm{ENH}$ à $\mathrm{pH} 9.1$ (valeur de $E_{\frac{1}{2}}$ de la vague III à ce $\mathrm{pH}$ ) puisque l'on devrait avoir

$$
E^{0}=\frac{1}{2}\left(E_{\mathrm{II}}^{0}+E_{\mathrm{III}}^{0}\right)
$$

or

$$
E^{0}=-0.36 \mathrm{~V} / \mathrm{ENH} \text { et } \frac{1}{2}\left(E_{\mathrm{II}}^{0}+E_{\mathrm{III}}^{0}\right)=-1.09 \mathrm{~V} / \mathrm{ENH}
$$

De même, si nous supposons que l'oxydation de A-A (pic $\mathrm{II}_{0}$ ) s'effectue selon

$$
\mathrm{A}-\mathrm{A} \rightleftarrows 2 \mathrm{~A}^{+}+2 e^{-} \text {à } E_{\mathrm{II}_{0}}^{0}=-0.11 \mathrm{~V} / \mathrm{ENH}
$$

la vague III ne peut correspondre à

$$
\mathrm{A}-\mathrm{A}+2 e^{-}+2 \mathrm{H}^{+} \rightarrow 2 \mathrm{AH} \text { à } E_{\mathrm{II}_{1}}^{0} \simeq-1.35 \mathrm{~V} / \mathrm{ENH} \text { à } \mathrm{pH} 9.1
$$

puisque l'on devrait avoir

$$
E^{0}=\frac{1}{2}\left(E_{\mathrm{III}}^{0}+E_{\mathrm{II}_{0}}^{0}\right)
$$

et que $\frac{1}{2}\left(E_{\mathrm{III}}^{0}+E_{\mathrm{II}_{0}}^{0}\right)$ est égal à $-0.73 \mathrm{~V} / \mathrm{ENH}$

Malgré le grand nombre de caractéristiques électrochimiques que nous avons recueilli sur l'iodure de $\mathrm{N}_{1}$-méthyl nicotinamide en solution queuse, il reste donc beaucoup d'indéterminations. En fait la multiplicité des résultats que nous avons obtenus a eu surtout une conséquence négative au niveau des interprétations. Cette multiplicité nous conduit ici à rejeter certains des mécanismes antérieurement proposés mais non démontrès, puisqu'ils conduisent à des combinaisons incohérentes. Les indéterminations qui apparaissent ainsi pourront certainement être levées en étudiant en détail le mécanisme des réactions correspondant aux vagues de réduction I et III et surtout aux vagues de réoxydation des produits de toutes les vagues de réduction. L'oxydation de A·, A-A, AH doit par exemple fournir un nouvel ensemble de mécanismes de réactions qui, ajouté aux mécanismes dès à présent connus, devrait permettre de dégager une interprétation de toutes les réactions d'oxydo-réduction relatives à ce composé modèle du $\mathrm{NAD}^{+}$.

\section{PARTIE EXPÉRIMENTALE}

\subsection{Réactifs}

Nous avons employé pour préparer les solutions tampons des réactifs de pureté analytique. Les concentrations des espèces tamponnant le $\mathrm{pH}$ sont au moins cinquante fois plus élevées que celle du réactif étudié : le plus souvent les concentrations des formes acides et basiques sont prises toutes deux égales à $0.5 \mathrm{M}$. La liste et composition des tampons utilisés est fournie dans le Tableau 1.

L’iodure de $\mathrm{N}_{1}$-méthyl nicotinamide utilisé, de provenance Sigma et K.\& K., est de pureté analytique. Son comportement polarographique n'a indiqué la présence d'aucune trace d'impureté électroactive.

Le mercure utilisé comme matériau d'électrode indicatrice est nettoyé très soigneusement peu de temps avant son emploi. Ce nettoyage comprend:

- une filtration sur papier filtre,

— un dégraissage en continu par le benzène ou l'éther de pétrole et rinçage à l'alcool et l'eau. 
TABLEAU 1

COMPOSITION DES SOLUTIONS UTILISÉES POUR ASSURER LE TRANSPORT DU COURANT ET FIXER LE pH.

\begin{tabular}{|c|c|c|c|c|c|c|}
\hline \multirow[t]{2}{*}{$p H$} & \multicolumn{2}{|l|}{ Acides } & \multicolumn{2}{|l|}{ Bases } & \multicolumn{2}{|c|}{ Ions neutres } \\
\hline & Nature & $\begin{array}{l}\text { Concn. } \\
M\end{array}$ & Nature & $\begin{array}{l}\text { Concn. } \\
M\end{array}$ & Nature & $\begin{array}{l}\text { Concn. } \\
M\end{array}$ \\
\hline $0.58 \pm 0.02$ & $\mathrm{HCl}$ & 0.50 & & & $\mathrm{Cl}^{-}$ & 0.50 \\
\hline $1.86 \pm 0.02$ & $\mathrm{H}_{3} \mathrm{PO}_{4}$ & 0.50 & $\mathrm{H}_{2} \mathrm{PO}_{4}^{-}$ & 0.50 & $\mathrm{~K}^{+}$ & 0.50 \\
\hline $2.41 \pm 0.05$ & ${ }^{+} \mathrm{H}_{3} \mathrm{NCH}_{2} \mathrm{CO}_{2} \mathrm{H}$ & 0.50 & ${ }^{+} \mathrm{H}_{3} \mathrm{NCH}_{2} \mathrm{CO}_{2}^{-}$ & 0.50 & $\mathrm{Cl}^{-}$ & 0.50 \\
\hline $3.56 \pm 0.02$ & $\mathrm{HCO}_{2} \mathrm{H}$ & 0.50 & $\mathrm{HCO}_{2}^{-}$ & 0.50 & $\mathrm{~K}^{+}$ & 0.50 \\
\hline $4.80 \pm 0.02$ & $\mathrm{CH}_{3} \mathrm{CO}_{2} \mathrm{H}$ & 0.50 & $\mathrm{CH}_{3} \mathrm{CO}_{2}^{-}$ & 0.50 & $\mathrm{~K}^{+}$ & 0.50 \\
\hline $5.78 \pm 0.02$ & di citrate & 0.50 & tri citrate & 0.50 & $\mathrm{~K}^{+}$ & 2.50 \\
\hline $6.68 \pm 0.05$ & $\mathrm{H}_{2} \mathrm{PO}_{4}^{-}$ & 0.50 & $\mathrm{HPO}_{4}^{2-}$ & 0.50 & $\mathrm{~K}^{+}$ & 1.50 \\
\hline $7.20 \pm 0.05$ & imidazole- $\mathrm{H}^{+}$ & 0.50 & imidazole & 0.50 & $\mathrm{Cl}^{-}$ & 0.50 \\
\hline $8.25 \pm 0.05$ & tris- $\mathrm{H}^{+}$ & 0.50 & tris & 0.50 & $\mathrm{Cl}^{-}$ & 0.50 \\
\hline $9.57 \pm 0.02$ & $\mathrm{H}_{3} \mathrm{BO}_{3}$ & 0.50 & $\mathrm{H}_{2} \mathrm{BO}_{3}^{-}$ & 0.50 & $\mathrm{~K}^{+}$ & 0.50 \\
\hline $9.82 \pm 0.02$ & $\mathrm{HCO}_{3}^{-}$ & 0.50 & $\mathrm{CO}_{3}^{2-}$ & 0.50 & $\mathrm{~K}^{+}$ & 1.50 \\
\hline $9.95 \pm 0.02$ & ${ }^{+} \mathrm{H}_{3} \mathrm{NCH}_{2} \mathrm{CO}_{2}^{-}$ & 0.50 & $\mathrm{H}_{2} \mathrm{NCH}_{2} \mathrm{CO}_{2}^{-}$ & 0.50 & $\mathrm{~K}^{+}$ & 0.50 \\
\hline
\end{tabular}

- un lavage en continu à $\mathrm{HNO}_{3} 5-10 \%$ et rinçage à l'eau distillée,

- une première distillation sous vide, mercure).

— une deuxième distillation sous vide plus poussé (pompe à diffusion de Le mercure est enfin conservé sous azote dans l'appareillage et nettoyé à nouveau dès l'apparition de traces d'impuretés à sa surface.

\subsection{Appareillage}

Polarographie à stillation commandée. L'ensemble de mesures utilisé comprend essentiellement des appareils Tacussel et un enregistreur Sefram. Il est composé de :

- un potentiostat PRT 500 LC imposant un potentiel défini entre une électrode de référence et une électrode indicatrice.

— un millivoltmètre électronique S6RZ à grande impédance d'entrée dont le signal de sortie est envoyé à la partie tension $(\mathrm{X})$ d'un enregistreur XY,

- un pilote de tension Servovit 9B permettant le balayage linéaire en fonction du temps du potentiel imposé par le potentiostat (vitesse réglable continûment entre 0.1 et $1100 \mathrm{mV} \min ^{-1}$ ),

- un marteau électrique MPO commandé par un générateur de commande de marteau à répétition GCMR qui fixe le temps de goutte continûment entre 0.01 et $100 \mathrm{~s}$.

L'enregistreur bigalvanométrique utilisé est un Luxytrace TR VAC Sefram branché pour sa partie courant $(\mathrm{Y})$ sur le circuit de l'électrode auxiliaire.

Cet ensemble expérimental permet le relevé direct de courbes intensitépotentiel avec une précision de mesure du courant de $1 \%$ et du potentiel de $\pm 2 \mathrm{mV}$.

Voltametrie cyclique sur disque immobile de carbone vitreux. L'ensemble de mesures utilisé pour la voltamétrie cyclique comprend essentiellement des appareils Tacussel et un oscilloscope Tetronix. Il est composé de: 
- un potentiostat à réponse rapide PRT 20-2X,

— un pilote de tension GSTP permettánt un balayage aller et retour, linéaire en fonction du temps, du potentiel imposé par le potentiostat.

- un millivoltmètre électronique S6RZ qui permet de mesurer avec précision le potentiel initial et l'amplitude du balayage,

- deux amplificateurs différentiels ADTP qui permettent de relier les voies $\mathrm{X}$ et $\mathrm{Y}$ de l'oscilloscope à la cellıle, sans modifier les potentiels et courants à mesurer.

L'enregistreur XY utilisé est un oscilloscope à mémoire Tektronix R 564 B muni de deux tiroirs amplificateurs.

Cet ensemble expérimental permet le relevé direct de courbes intensitépotentiel avec une précision de mesure du courant de $5 \%$ et du potentiel de $\pm 10 \mathrm{mV}$.

Cellules électrochimiques et électrodes. Nous avons utilisé des cellules, de type classique à trois électrodes, de volume de travail compris entre 3 et $15 \mathrm{ml}$. Une double paroi permet, grace à une circulation d'eau, de maintenir constante la température de la cellule. L'oxygène dissous dans les solutions est éliminé au moyen d'un barbotage d'azote de qualité R utilisé sans purification supplémentaire. Après une quinzaine de

TABLEAU 2

CARACTÉRISTIQUES DU CA PILLAIRE UTILISÉ POUR LA POLAROGRAPHIE À STILLATION COMMANDÉE ET POUR LA VOLTAMÉTRIE CYCLIQUE SUR GOUTTE DE MERCURE

\begin{tabular}{lllll}
\hline No & $\begin{array}{l}\text { Longueur } \\
/ \mathrm{cm}\end{array}$ & $\begin{array}{l}\text { Hauteur de } \\
\text { mercure }\end{array}$ & $\begin{array}{l}\text { Temps de goutte } \\
\text { libre à } 25^{\circ} \mathrm{C}^{b} / \mathrm{s}\end{array}$ & $\begin{array}{l}\text { Débit de mercure }{ }^{b} \\
/ \mathrm{mg} \mathrm{s}^{-1}\end{array}$ \\
\hline \multirow{2}{*}{20} & 25 & $12.0-12.8$ & 0.63 \\
& 35 & $8.5-10.0$ & 0.92 \\
& 45 & $6.6-7.4$ & 1.18 \\
& 55 & $5.4-6.0$ & 1.45 \\
& 65 & $4.8-4.9$ & 1.72 \\
& 72.5 & 4.6 & 1.92 \\
\hline
\end{tabular}

\footnotetext{
${ }^{a}$ Hauteurs de mercure, non corrigées, au-dessus de l'extrémité inférieure du capillaire.

${ }^{b}$ Temps de goutte libre et débits de mercure ont été mesurés en circuit ouvert, le capillaire étant plongé dans de l'eau permutée.
}

minutes de barbotage, un courant d'azote est maintenu au-dessus de la solution pendant toute la durée des expériences.

Les électrodes indicatrices sont constituées suivant la nature de l'étude:

- soit d'un capillaire à goutte de mercure, de temps de goutte libre compris entre 4 et $15 \mathrm{~s}$ et pour lequel on fixe mécaniquement le temps de goutte à une valeur inférieure, généralement $0.50 \mathrm{~s}$. Cette régulation mécanique élimine les variations et irrégularités du temps de goutte à potentiel très négatif. Le Tableau 2 donne les caractéristiques de ces capillaires, temps de goutte libre et débit pour les hauteurs de mercure utilisées.

- soit d'un disque de carbone vitreux de $1.2 \mathrm{~mm}$ de diamètre.

Bien que des électrodes de référence $\mathrm{Ag} / \mathrm{AgCl}, \mathrm{KCL}$ saturé (Tacussel type $\mathrm{AgCl}$ 10) aient été généralement utilisées, tous les potentiels cités sont rapportés à l'électrode normale à hydrogène à $25.0 \pm 0.2^{\circ} \mathrm{C}$. Le potentiel fourni par les électrodes de référence 
est périodiquement contrôlé avec des électrodes témoins: ce potentiel est connu avec une incertitude inférieure à $1 \mathrm{mV}$. Pour limiter la diffusion d'ions gênants à l'intérieur de l'électrode de référence, celle-ci a été séparée de la solution par un compartiment fritté rempli d'une solution saturée en chlorure de potassium.

\subsection{Mode opératoire}

Pour chaque $\mathrm{pH}$ étudié un stock de solution tampon est préparé (Tableau 1) à partir de réactifs de pureté analytique. Une quantité connue $(5 \mathrm{ml})$ de cette solution est placée dans la cellule électrochimique. Un premier tracé polarographique ou voltamétrique est effectué dans cette solution plus ou moins saturée d'air suivi d'un deuxième tracé, effectué a près avoir chassé l'oxygène en faisant barboter de l'azote pendant une quinzaine de minutes. Les solutions de réactif étudié sont obtenues en ajoutant dans ce volume de solution tampon des volumes connus de solutions aqueuse concentrées de réactif $(0.50$ et $0.050 \mathrm{M})$. Cette addition est généralement réalisée à l'aide d'une microburette à piston Manostat Digi-Pet. Le $\mathrm{pH}$ de la solution est mesuré avec une électrode de verre combinée (Tacussel type TCBC 101) et un pH-mètre electronique (Tacussel type TS 70) avant et après cette addition et les opérations de barbotage d'azote; ceci permet de vérifier que la variation du $\mathrm{pH}$ au cours des manipulations reste inférieure à quelques centièmes d'unité de $\mathrm{pH}$.

En polarographie les courants limites de vague ont été relevés sur les tracés intensité-potentiel suivant les méthodes proposées par Zuman ${ }^{33}$ soit individuellement pour chaque vague, soit additivement. Lorsque cela représente une correction notable, c'est-à-dire pour les concentrations les plus faibles en réactif, le courant de fond obtenu en présence de solution tampon seule est soustrait du courant obtenu en présence de réactif.

RÉSUMÉ

Nous avons étudié la réduction électrochimique de solutions aqueuses d'iodure de $\mathrm{N}_{1}$-méthyl nicotinamide en utilisant essentiellement la polarographie à stillation commandée. Aux pH inférieurs à 3 nous avons relevé deux vagues (I et I'), la deuxième présentant un courant anormalement élevé. Aux pH compris entre 3 et 10, la vague I est remplacée par deux vagues de hauteur sensiblement moitié : la première vague (II) ne dépend pas du $\mathrm{pH}$, tandis que le potentiel de la deuxième (III) diminue de $60 \mathrm{mV}$ lorsque le $\mathrm{pH}$ augmente d'une unité. L'influence sur le potentiel de demi-vague de la concentration de réactif et du temps de goutte imposé montre, en accord avec les résultats de l'analyse mathématique de la forme de la vague II, que celle-ci correspond en solution diluée à la fixation d'un électron par molécule de réactif pris à la dimérisation du radical neutre formé. La réoxydation électrochimique de ce dimère a été réalisée aux pH compris entre 6 et 10 par voltamétrie cyclique sur disque de carbone vitreux : son potentiel de dépend pas du pH et est situé $740 \mathrm{mV}$ au-dessus du potentiel de la vague II.

\section{SUMMARY}

The reduction behaviour of $\mathrm{N}_{1}$-methylnicotinamide iodide has been studied by drop-time controlled polarography. In the $\mathrm{pH}$ range $0-3$, two $\mathrm{pH}$-dependent waves 
were observed (I and $\mathrm{I}^{\prime}$ ) the latter having an abnormally high limiting current. In the $\mathrm{pH}$ range 3-10, wave I splits into two waves (II and III) of similar height, the former being $\mathrm{pH}$-independent whereas the half-wave potential of the latter shifts $-60 \mathrm{mV}$ per $\mathrm{pH}$ unit. The concentration and drop-time dependence of the half-wave potential of wave II, in accordance with the logarithmic analysis of its shape, shows that it corresponds, in dilute solutions, to a monoelectronation followed by a dimerisation of the neutral free radical formed. In the $\mathrm{pH}$ range 6-10 reoxidation of this dimer was obtained at $740 \mathrm{mV}$ above the potential of wave II, by cyclic voltammetry on stationary vitreous carbon electrodes. This reoxidation peak is also $\mathrm{pH}$-independent.

\section{BIELIOGRAPHIE}

1 D. Thévenot et G. Hammouya, Experientia, Suppl., 18 (1971) 631.

2 D. Thévenot et R. Buvet, J. Electroanal. Chem., 39 (1972) 429.

3 D. Thévenot et G. Hammouya, Experientia, Suppl., 18 (1971) 647.

4 L. Meites, Polarographic Techniques, Interscience, New York, 2nd ed., 1965, pp. 288-289.

5 C. P. Andrieux, L. Nadjo et J. M. Savéant, J. Electroanal. Chem., 26 (1970) 147.

6 P. Zuman dans P. Zuman et I. M. Kolthoff (Eds.), Progress in Polarography, Vol II, Interscience, New York, 1962, pp. 583-600.

7 G. Charlot et R. Gauguin, Les Méthodes d' Analyse des Réactions en Solution, Masson, Paris, 1951, p. 124.

8 G. Charlot, J. Badoz-Lambling et B. Trémillon, Les Réactions Électrochimiques, Masson, Paris, 1959, p. 116.

9 I. M. Kolthoff et J. J. Lingane, Polarography, Vol. I, Interscience, New York, 1952, p. 202.

10 L. Meites, Polarographic Techniques, Interscience, New York, 1965, pp. 228-229.

11 S. G. Mairanovskii dans P. Zuman (Ed.), Catalytic and Kinetic Waves in Polarography, Plenum Press, New York, 1968, p. 216.

12 I. M. Kolthoff et J. J. Lingane, Polarography, Vol. I, Interscience, New York, 2nd ed., 1952, pp. 90-93.

13 L. Meites, Polarographic Techniques, Interscience, New York, 2nd ed., 1965, pp. 138-140.

14 S. G. Mairanovskii dans P. Zuman (Ed.), Catalytic and Kinetic Waves in Polarography, Plenum Press, New York, 1968, p. 12 et pp. 85-93.

15 S. G. Mairanovskii, Dokl. Akad. Nauk SSSR, 110 (1956) 593.

16 J. Volke, M. Naarova et V. Volkova, 21st C.I.T.C.E. Meeting, Prague, 1970 extended abstr., p. 33.

17 J. M. Savéant et E. Vianello, C.R., 256 (1963) 2597.

18 W. Kemula, Z. Grabowski et M. Kalinowski, Naturwiss., 47 (1960) 514.

19 S. G. Mairanovskii et V. N. Pavlov, Zh. Fiz. Khim., 38 (1964) 1804.

20 C. P. Andrieux et J. M. Savéant, J. Electroanal. Chem., 26 (1970) 223.

21 J. Koutecky et V. Hanus, Collect. Czech. Chem. Commun., 20 (1955) 124.

22 S. G. Mairanovskii dans P. Zuman (Ed.), Catalytic and Kinetic Waves in Polarography, Plenum Press, New York, 1968, pp. 227-239.

23 M. L. Olmstead, R. G. Hamilton et R. S. Nicholson, Anal. Chem., 41 (1969) 260.

24 M. L. Olmstead et R. S. Nicholson, Anal. Chem., 41 (1969) 851.

25 C. P. Andrieux, Thèse, Paris, 1971.

26 L. Nadjo, Thèse, Paris, 1971.

27 R. N. Adams, Electrochemistry at Solid Electrodes, Marcel Dekker, New York, 1969, p. 126.

28 J. Nakaya, Nippon Kagaku Zasshi, 81 (1960) 1459.

29 W. Ciusa, P. M. Strocchi et G. Adamo, Giazz. Chim. Ital., 80 (1950) 604.

30 S. J. Leach, J. H. Baxendale et M. G. Evans, Aust. J. Chem., 6 (1953) 395.

31 J. N. Burnett et A. L. Underwood, J. Org. Chem., 30 (1965) 1154.

32 A. J. Cunningham et A. L. Underwood, Biochem., 6 (1967) 266.

33 P. Zuman dans P. Zuman et I. M. Kolthoff (Eds.), Progress in Polarography Vol. II, Interscience, New York, 1962, pp. 583-600.

34 F. J. Land et A. J. Swallow, Biochim. Biophys. Acta, 162 (1968) 327.

35 D. Thévenot et R. Bubet, J. Electroanal. Chem., 39 (1972) 429.

36 P. Leduc et $\mathrm{D}$. Thévenot, résultats non publiés. 
